\title{
Enhancement of charge transfer in thermally-expanded and strain-stabilized TIPS-pentacene thin films
}

\author{
Yang Li (1) and Jing Wan \\ Materials Science Program, University of Vermont, Burlington, Vermont 05405, USA \\ Detlef-M. Smilgies \\ Cornell High Energy Synchrotron Source, Cornell University, Ithaca, New York 14853, USA \\ Richards Miller \\ Department of Physics, University of Vermont, Burlington, Vermont 05405, USA \\ Randall L. Headrick (i)* \\ Department of Physics and Materials Science Program, University of Vermont, Burlington, Vermont 05405, USA
}

(Received 5 April 2020; revised 13 July 2020; accepted 5 August 2020; published 24 August 2020)

\begin{abstract}
We present an extensive study of the optical and electronic properties of TIPS-pentacene thin films utilizing in situ x-ray diffraction, polarized optical spectroscopy, and $a b$ initio density functional theory. The influence of molecular packing on the properties are reported for thin films deposited in the temperature range from $25^{\circ} \mathrm{C}$ to $140^{\circ} \mathrm{C}$ and for films that are strain stabilized at their as-deposited lattice spacings after cooling to room temperature. Anisotropic thermal expansion causes relative displacement of neighboring molecules while maintaining a nearly constant stacking distance. This leads to a large blueshift in the absorption spectrum as the temperature increases. The blueshift largely reverses a redshift at room temperature compared to the solution absorption spectrum. A reduction in the ratio of the first two vibronic peaks relative to the solution spectrum is also observed. This combination of electronic and vibronic effects is a signature of charge transfer excitonic coupling with a positive coupling constant $J_{\mathrm{CT}}$, which depends sensitively on the alignment of the nodes of the frontier molecular orbitals with those on neighboring molecules. These effects are also correlated with the sign and magnitude of electron and hole charge transfer integrals $t_{e}$ and $t_{h}$ calculated from density functional theory that provide additional evidence for charge transfer mediated coupling, as well as insight into the origin of an experimentally observed enhancement of the field-effect transistor mobility in strain-stabilized thin films. The results suggest approaches to improve carrier mobility in strained thin films and for optical monitoring of electronic changes.
\end{abstract}

DOI: 10.1103/PhysRevResearch.2.033294

\section{INTRODUCTION}

Two of the most critical experimentally accessible properties of small-molecule organic semiconductor materials are the charge carrier mobility, which probes charge transport, and the optical absorption spectrum which probes the energy levels of excited states. A deep understanding of how the structure affects these properties is a key challenge [1,2]. It is vital to understand the fundamental mechanisms of carrier transport in order to design new materials that will lead to improved organic thin film transistors with faster operation and lower power consumption in advanced liquid crystal and

\footnotetext{
*rheadrick@uvm.edu

Published by the American Physical Society under the terms of the Creative Commons Attribution 4.0 International license. Further distribution of this work must maintain attribution to the author(s) and the published article's title, journal citation, and DOI.
}

organic light-emitting displays [3-5]. In the case of optical absorption, the spectrum gives important insight into the types of excitations present.

There have been great improvements in the charge carrier mobility of $\pi$-conjugated organic semiconductors over the last 30 years as new materials with improved properties have been identified [6-10]. These molecular solids typically have small $\pi-\pi$ stacking distances around $3.5 \AA$, which promotes charge transfer and delocalization [11,12]. Charge transport has previously been assumed to be through hopping of localized carriers since the semiclassical mean-free-path of carriers is found to be less than the intermolecular distance [13]. However, recent experiments reveal carrier transport characterized by bandlike mobility, that is, increasing mobility as the temperature decreases, implying that charge carriers are delocalized [14,15]. This ambiguous localized/delocalized behavior has spurred the adoption of a new paradigm, where dynamic disorder caused by molecular thermal vibrations is sufficient to break the translational symmetry of the electronic Hamiltonian, producing transient localization of the charge 
carriers while maintaining coherence over a characteristic length scale $L$ on the order of the molecular spacing [16-19]. These new insights suggest ways to increase $L$ by (i) reducing the sensitivity of the intermolecular electronic coupling to thermal vibrations and (ii) designing materials that have reduced dynamic disorder [18,19].

Excitons in organic semiconductors can be tightly bound to a single molecule (Frenkel type) or they can be composed of charges separated by one or a few molecular spacings (charge transfer type). In many previous studies, an energy shift in the optical absorption spectra of certain organic semiconductors is found to be highly sensitive to small changes of the molecular packing $[2,20]$. This effect, termed "crystallochromy," occurs when the energy difference between the Frenkel exciton and charge transfer (CT) excited states is small, so that neutral molecular excitations and charge transfer excitations mix via electron and hole transfer [12,21-23]. The interference between these states produces a short-range coupling that results in a significant shift in the absorption bands relative to the (highly localized) Frenkel exciton [22-26]. The sensitivity to the crystal packing arises since these effects depend on the overlap of the nodal patterns of the highest occupied molecular orbital (HOMO) and lowest unoccupied molecular orbital (LUMO) of neighboring molecules, which is sensitive to sub-angstrom relative motion. The excitation energy shift caused by CT/Frenkel exciton mixing can be characterized by the magnitude of the hole and electron charge transfer integrals $t_{h}$ and $t_{e}$.

The impact of molecular packing on the optical and charge transport properties are often treated separately. However, these effects are actually linked at a fundamental level, and it is of interest to understand the interrelationship between them, as well as how they respond to strain and thermal expansion. In this paper, we highlight the fundamental relationship between these two phenomena in 6,13Bis(triisopropylsilylethynyl)pentacene (TIPS-pentacene).

The structure of this paper is as follows: In Sec. III A, we address the basic structural and electronic properties of TIPS-pentacene thin films. These include the crystallographic orientation of twin grains [27,28] and the directions of transition dipoles relative to the molecular axis [29,30]. Although there is existing literature on these effects, we examine them in detail in order to clarify the correct structure-property relationships. In Sec. III B, we show that the large thermal expansion of TIPS-pentacene leads to an enhancement of the hole transport in strain-stabilized thin films and that it modulates the charge transfer integrals and their sensitivity to dynamic disorder. In Sec. III C, we show that the optical excitation energies can also be tuned by thermal expansion effects and we investigate how this is also related to the change of the theoretically-determined charge transfer integrals.

\section{EXPERIMENTAL}

TIPS-pentacene $(\geqslant 99 \%)$ was purchased from Sigma Aldrich. All TIPS-pentacene thin films were prepared by the hollow capillary pen-writer method [28,31] except the samples B2 and B3 that were used for mobility characterization. Our hollow rectangular capillary has a size of $0.5 \mathrm{~mm} \times$ $5.0 \mathrm{~mm}$ I.D. (Wale apparatus Co. 4905-100) thus it can only be utilized to write narrow films with a width around $5 \mathrm{~mm}$.
The glass substrates (fused silica corning 7980 of UV grade) we used for making transistors were pre-diced to a standard substrate size of $15 \mathrm{~mm} \times 20 \mathrm{~mm}$ and polished both sides with a roughness smaller than $0.7 \mathrm{~nm}$. For transistor arrays, a custom-designed slot writer with a slot size of $0.5 \mathrm{~mm} \times$ $20 \mathrm{~mm}$ was used to deposit TIPS-pentacene films to make the film fully cover the substrate and gold contacts, as described below. Film thickness was measured for each sample by a Dektak XT stylus profilometer. The experimental conditions for all samples are summarized in Table I and they are individually described below.

Sample A1 was deposited at $90{ }^{\circ} \mathrm{C}$ with a low writing speed of $0.05 \mathrm{~mm} / \mathrm{s}$ in order to obtain highly oriented grains. In situ microbeam grazing incidence wide-angle $\mathrm{x}$-ray scattering ( $\mu$ GIWAXS) was carried out at the Cornell High Energy Synchrotron Source (CHESS), beamline D1. The X-ray incidence direction in each case was perpendicular to the film's writing direction. This data was used to study the molecular orientation of the grains. Polarized optical microscopy was performed using a Zeiss microscope (Axioskop 40) after the sample was cooled to room temperature.

Sample A2 was deposited on a glass slide to collect polarized absorption spectra. The film was deposited at a low writing speed $(0.08 \mathrm{~mm} / \mathrm{s})$ from a $44 \mathrm{mg} / \mathrm{ml}$ toluene solution to get large oriented grain size $(>100 \mu \mathrm{m})$. The film was relatively thick $(620 \mathrm{~nm})$ so that it would have strong absorbance. A schematic setup of the single-grain polarization spectroscopy is shown in Fig. 1(a). Briefly, the setup is as follows: A thin film on a glass substrate is placed in a temperature controlled microscope stage (Linkam THMS600) which is mounted on a rotation stage, and a fixed polarizer is placed between the light source and the sample. An optical microscope (Olympus BXFM) with an integrated UV-Vis spectrometer (Angstrom Sun Technologies Inc.) is used to acquire absorption spectra within a single grain [Figs. 1(b) and 1(c)]. Polarized absorption spectra were collected at each angle over the range $-30^{\circ}$ and $60^{\circ}$ with respect to the grain boundary in order to map out the polarization dependence of the absorption along both the short and long axes of the TIPS-pentacene molecular core.

$\mu$ GIWAXS was carried out in an in situ study at CHESS to determine the thermal expansion induced structure change of polycrystalline thin films. The samples B1a and B1k were made at $25^{\circ} \mathrm{C}$ and $134^{\circ} \mathrm{C}$, respectively, and their $\mathrm{x}$-ray data were collected at the deposition temperature of each sample in order to avoid measurable strain effects.

To study the charge transport properties, TIPS-pentacene thin films deposited at $25^{\circ} \mathrm{C}$ and $130{ }^{\circ} \mathrm{C}$ (samples B2 and B3) were used as active layers for organic field-effect transistors. The glass slides were first ultrasonic cleaned in deionized water, acetone, and isopropanol for $10 \mathrm{~min}$ and then the phenyltriethoxysilane (PTES) treatment was accomplished by immersing the cleaned glass slides into a toluene solution of $3 \mathrm{wt} \%$ PTES and heated to $110^{\circ} \mathrm{C}$ for $15 \mathrm{~h}[32,33]$. After PTES treatment, the substrates were rinsed in toluene, acetone and isopropanol for $1 \mathrm{~min}$. Gold contacts $(30 \mathrm{~nm})$ were evaporated using a shadow mask to form arrays of source and drain electrodes with a channel length of $30 \mu \mathrm{m}$ and a channel width of $1000 \mu \mathrm{m}$. These contacts were then treated for $20 \mathrm{~min}$ using $30 \mathrm{mM}$ solution of pentafluorobenzene thiol 
TABLE I. Deposition conditions of TIPS-pentacene thin films discussed in Sec. III.

\begin{tabular}{|c|c|c|c|c|c|c|c|}
\hline Samples & Figure & $\begin{array}{c}\text { Temp. } \\
\left({ }^{\circ} \mathrm{C}\right)\end{array}$ & $\begin{array}{l}\text { Concentration } \\
(\mathrm{mg} / \mathrm{ml})\end{array}$ & $\begin{array}{l}\text { Speed } \\
(\mathrm{mm} / \mathrm{s})\end{array}$ & Substrate & Solvent & $\begin{array}{c}\text { Thickness } \\
\text { (nm) }\end{array}$ \\
\hline A1 & 2 & 90 & 1.5 & 0.05 & $\mathrm{Si} / \mathrm{SiO}_{2}$ & Mesitylene & 210 \\
\hline $\mathrm{A} 2$ & $1,3,4,5$ & 25 & 44 & 0.08 & glass & Toluene & 620 \\
\hline B1a & 6 & 25 & 1.5 & 25 & $\mathrm{Si} / \mathrm{SiO}_{2}$ & Toluene & 85 \\
\hline B $1 \mathrm{k}$ & 6 & 134 & 1.5 & 25 & $\mathrm{Si} / \mathrm{SiO}_{2}$ & Mesitylene & 106 \\
\hline $\mathrm{B} 2$ & 7,8 & 25 & 20 & 0.05 & glass & Toluene & 25 \\
\hline B3 & 7,8 & 130 & 10 & 0.6 & glass & Mixed solvent & 30 \\
\hline $\mathrm{C} 1$ & 10,11 & 25 & 25 & 0.02 & glass & Toluene & 650 \\
\hline
\end{tabular}

(PFBT) in isopropanol followed by a 2 min rinse in pure isopropanol and a $15 \mathrm{~min}$ annealing at $60^{\circ} \mathrm{C}$. The TIPSpentacene form I film (sample B2) was deposited from pure toluene solution and form II film (sample B3) was prepared from a blend of dichlorobenzene and mesitylene with a volume ratio of $4: 1$. The deposition speed, concentration, and TIPS-pentacene film thickness information can be found in Table I. The TIPS-pentacene film was annealed at $100{ }^{\circ} \mathrm{C}$ for 10 min then a CYTOP layer was prepared by slot writing from a 809M CYTOP solution using CT-SOLV100E as solvent (AGC Chemicals Co.). The volume ratio of 809M CYTOP to its solvent is $1: 1.5$ and the deposition speed is $4 \mathrm{~mm} / \mathrm{s}$. The (a)

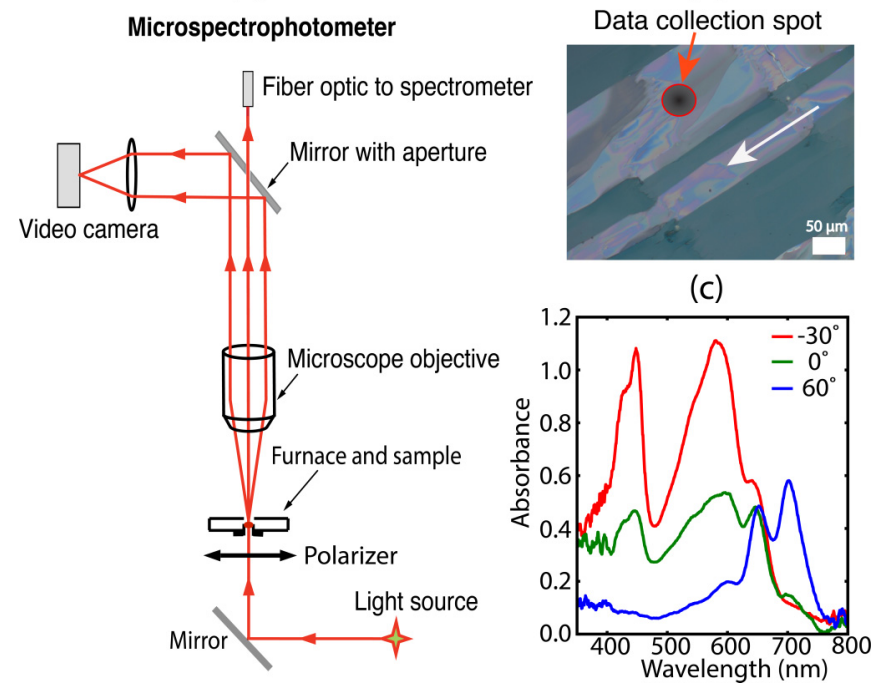

FIG. 1. (a) Schematic of the setup for polarized transmission spectrum collection. A polarizer is placed under a rotational sample stage and the polarization is put along the writing direction. A mirror with an aperture is inserted into the light path to select a small area of interest. (b) The polarized optical image of a sample deposited at $0.08 \mathrm{~mm} / \mathrm{s}$ at $25^{\circ} \mathrm{C}$ on glass substrate (sample A2). The large grain size enables us to put the transmission data collection spot in a single grain since the data collection spot is about $50 \mu \mathrm{m}$ in diameter under 20 times magnification lens. The white arrow indicates the writing direction of the film. The angle was set to be $0^{\circ}$ when the polarization was along the writing direction. (c) Typical polarized absorption spectra. Red: The sample was rotated by $-30^{\circ}$ to the writing direction. Green: The sample was placed at $0^{\circ}$. Blue: The sample was rotated by $60^{\circ}$. sample was annealed at $100{ }^{\circ} \mathrm{C}$ for $20 \mathrm{~min}$, resulting in a 1100 $\mathrm{nm}$ thick CYTOP layer with a capacitance of $1.7 \mathrm{nF} / \mathrm{cm}^{2}$. The TIPS-pentacene and CYTOP film deposition and annealing were carried out in a nitrogen atmosphere. Finally a 100 $\mathrm{nm}$ thick aluminum layer was thermally evaporated using a shadow mask in a bell jar to serve as the gate electrode. For each sample, 14-15 transistors were characterized.

The intermolecular electronic couplings between the highest occupied and lowest unoccupied molecular orbitals ( $t_{\text {homo }}$ and $t_{\text {lumo }}$ ) of TIPS-pentacene dimers are calculated using the ADF (Amsterdam density functional) package [34]. The PW91 [35] exchange-correlation function and the TZP basis set are used in the calculation for both dimer I ([100] direction) and dimer II ([110] direction). The sign of electronic coupling ( $t_{\text {homo }}$ and $t_{\text {lumo }}$ ) is determined by the translational symmetry of the molecular orbitals of the dimer. The electron charge transfer integral is obtained by $t_{e}=t_{\text {lumo, }}$, and the hole charge transfer integral is obtained by $t_{h}=-t_{\text {homo }}$.

$\mathrm{X}$-ray diffraction data were collected at $23^{\circ} \mathrm{C}$ for a TIPSpentacene single crystal using a Bruker Apex II CCD singlecrystal diffractometer. This data was used to obtain the full structure of the room temperature phase. The unit cell parameters of the TIPS-pentacene high temperature phase were obtained at $134{ }^{\circ} \mathrm{C}$ from the in situ $\mu$ GIWAXS on the B1k thin film sample. We did not obtain the full structure from the $\mathrm{x}$-ray data in this case since it was not possible to measure enough reflections from the thin film to obtain a reliable structure. Instead a geometry optimization with the lattice constants constrained to the experimentally measured values was performed using the BAND program with PBE functional and D3 dispersion correction with Becke-Johnson dampling PBE-D3(BJ). The TZP basis set was used in the geometry optimization.

To study the influence of the thermal expansion on the optical properties, Sample C1 was deposited on a glass slide at $0.02 \mathrm{~mm} / \mathrm{s}$ in order to obtain large grain size. The temperature dependence of single-grain polarized spectra were collected using the setup in Fig. 1. The sample was $650 \mathrm{~nm}$ thick. Such large thickness films can expand and contract freely without being significantly influenced by the substrate during annealing up to $140^{\circ} \mathrm{C}$ and subsequent cooling. The heating and cooling rate was $2{ }^{\circ} \mathrm{C} / \mathrm{min}$ for these temperature scans, and temperature steps of $10^{\circ} \mathrm{C}$ were carried out. The temperature was maintained at each step for about 10 mins and polarized absorption spectra were collected at $-30^{\circ}$ and $60^{\circ}$ with respect to the grain boundary. 
(a)

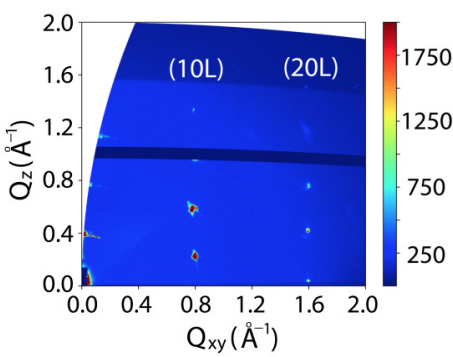

(c)

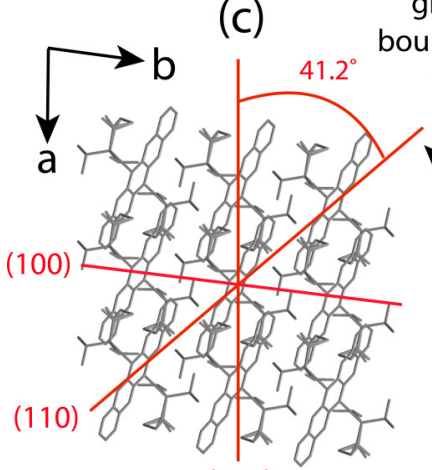

(010) (b)

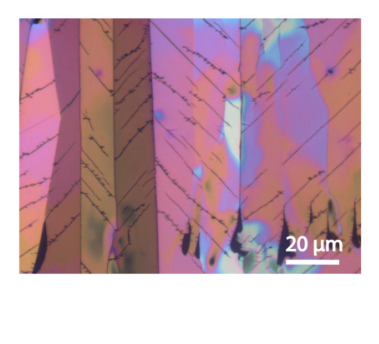

$\begin{array}{ll}\text { grain } & (d)\end{array}$

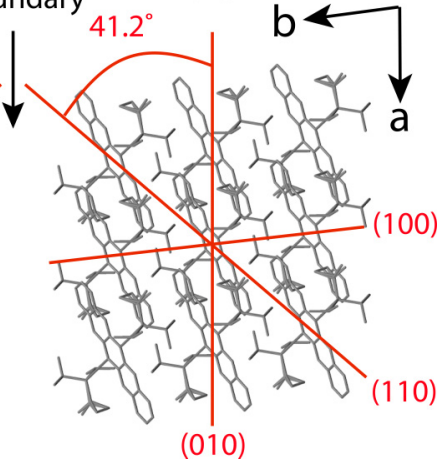

FIG. 2. (a) A typical x-ray scattering image for an aligned TIPSpentacene film deposited at $90{ }^{\circ} \mathrm{C}$ (sample A1 in Table I). In situ $\mathrm{X}$-ray scattering was carried out at the deposition temperature, and the x-ray beam was perpendicular to the writing direction. (b) A polarized optical image of the sample taken after it was cooled to room temperature. V-shaped cracks are formed and the angle between the cracks and twin boundary is about $40^{\circ}$. (c),(d) A model of twin grains formed in TIPS pentacene thin films. The twin boundary is along the $a$ axis which also corresponds to the pen-writing direction.

\section{RESULTS AND DISCUSSION}

\section{A. Molecular packing geometry and transition dipole directions}

In this section, we determine the crystalline orientation of aligned TIPS-pentacene films and the directions of transition dipoles relative to the molecular axes. This information is crucial for understanding the structure-property relationships.

\section{Molecular packing geometry}

In situ X-ray scattering data for sample A1 is shown in Fig. 2(a) which was collected at the deposition temperature. The x-ray beam direction is perpendicular to the writing direction of our film and only (10L) and (20L) are observed, indicating that the $\mathrm{x}$-ray beam is incident at a small angle to the (100) crystalline plane. A polarized optical image of the sample is shown in Fig. 2(b). The image was taken after the sample was cooled to room temperature. Cracks that had formed during cooling are visible in the image. This sample was intentionally fabricated with a relatively large film thickness $(210 \mathrm{~nm})$ in order to induce cracking during the cooling process, so that we can determine the orientation of the dominant cracks. In all of our aligned TIPS-pentacene thin films, the grain boundaries of the film are parallel to the writing direction.

The molecular orientation within the grains is illustrated in Figs. 2(c) and 2(d) based on a model that we have proposed in previous work [28]. In this model, the grain boundaries are twins oriented along the $a$ axis of the unit cell. The molecular orientation within one grain can be obtained by rotating the crystal structure of a neighboring twin by an angle of $180^{\circ}$ about an axis perpendicular to the boundary. In Figs. 2(c) and 2(d), the angle between the $a$ axis and the long axis of the molecule is $28^{\circ}$ (calculated using the supplemental structure file of the room temperature phase) [36]. This is consistent with Fig. 2(a) that the twin boundary is along the [100] direction. This conclusion is also supported by evidence from several other groups [37,38].

The cracking pattern is another important clue to help determine the molecular orientation. The cracks are V-shaped and the angle between the cracks and grain boundary in Fig. 2(b) is found to be $40^{\circ}$, which is very close to the orientation of (110) planes [see Fig. 2(c)]. A similar orientation of cracks has previously been observed [27,29]. We find that the (110) $d$ spacing undergoes the largest change during cooling, so the cracks tend to occur along these planes. For very thick films (not shown) cracks often occur along other directions in addition to the (110) oriented cracks [27,33,39].

\section{Direction of transition dipoles}

We can also correlate the packing geometry with its polarization-dependent optical absorbance. Here we use single-crystalline-grain polarized absorption spectra to determine the transition dipole directions. The data collection spot is put within a single grain as shown in Fig. 1(b). When the polarization direction is along the twin boundary we define the angle to be $\theta=0^{\circ}$. The sample is rotated in steps of $10^{\circ}$ to collect the polarized absorption spectra. Thirty-six polarized absorption spectra were collected in total, and ten of them are plotted in Fig. 3(a). The peaks are labeled as 1, 2, 3, 4, 5, and 6 starting from the long wavelength side [30]. In order to get the absorbance values of all six peaks, the data is fitted with a Lorentz oscillator model [40]. Examples of the fitting curves are given in Supplemental Material Fig. S1 and all the fitting results are listed in Table $\mathrm{S} 1$ [36]. The absorbance data is fitted by the equation [41]:

$A(\theta)=-\log _{10}\left(10^{-A_{\min }} \sin ^{2}\left(\theta-\theta_{0}\right)+10^{-A_{\max }} \cos ^{2}\left(\theta-\theta_{0}\right)\right)$.

The fitting parameters $A_{\max }, A_{\min }$, and $\theta_{0}$ are given in Supplemental Material Table S1 for all six peaks [36]. An example of the fitted data for peak 1 is shown in Fig. 3(c), and the complete series is shown in Fig. 4. The angle $\theta_{0}$ is the angle that gives the maximum absorbance. We find that peak 1 becomes strongest when the rotation angle is $60^{\circ}$, whereas peaks 3 and 5 become strongest when the rotation angle is $-30^{\circ}$. As we discussed in the last section, the angle between the long axis of the molecule and the crystallographic $a$ axis is $-28^{\circ}$ and the angle between the short axis of the molecule and the $a$ axis is $62^{\circ}$. These results indicate that the transition dipole for peak 1 , which corresponds to the singlet $S_{0}-S_{1}$ transition, is along the molecular short axis.

The observation that the transition dipole of peak 1 is along the short axis of the molecule can be justified from selection rules based on the symmetry of the molecular orbitals. 
(a)

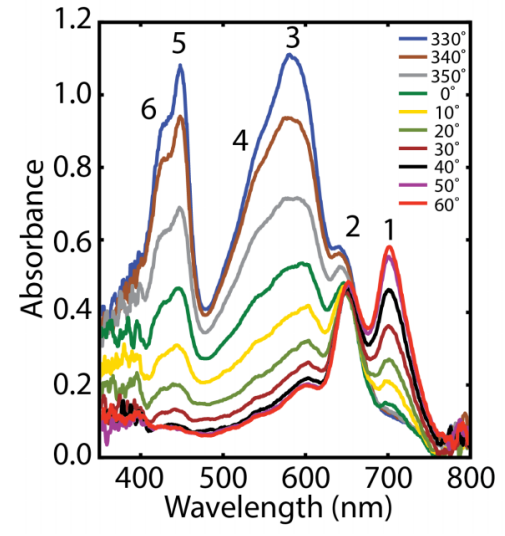

(b)

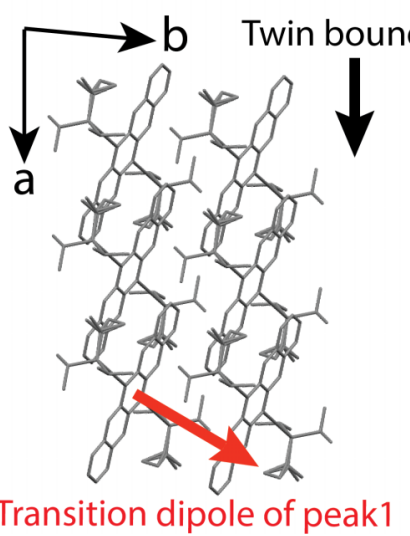

(c)

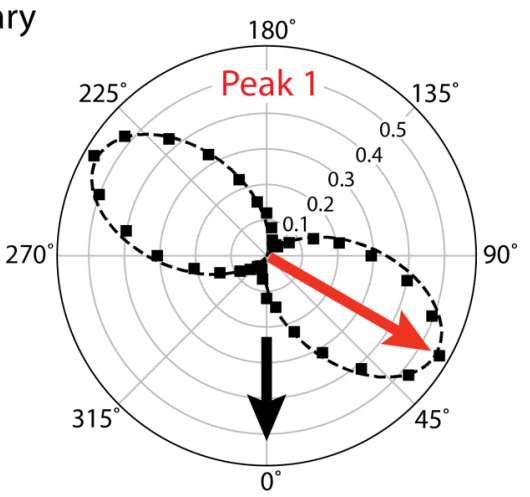

FIG. 3. (a) Typical single grain polarized absorption spectra when the sample is rotated from $-30^{\circ}$ to $60^{\circ}$ respect to the twin boundary with a step of $10^{\circ}$. From the low energy to the high energy side, we labeled the absorption peaks as 1, 2, 3, 4, 5, and 6. (b) Schematic of TIPS-pentacene molecule structure plotted using the supplemental structure file for the room temperature phase, top view. [36] At $0^{\circ}$, the twin boundary is along the polarized direction. The red arrow indicates the direction of transition dipole of peak 1 . Note that its direction is in the plane of the molecule and it has a small component in the plane of the film due to the tilt of the molecule along its long axis. (c) Angle dependence of absorbance of peak 1 . This sample is sample A2 in Table I.

Unsubstituted pentacene has $D_{2 h}$ symmetry and its HOMO and LUMO states belong to the $B_{1 g}$ and $B_{2 u}$ group representations, respectively. The selection rules determine that the HOMO to LUMO transition is only allowed along the short axis for the molecule. The TIPS-pentacene molecule belongs to the lower symmetry $C_{i}$ point group due to the fact that it has two side groups. The side groups and the lower symmetry of the crystalline environment break the selection rule for

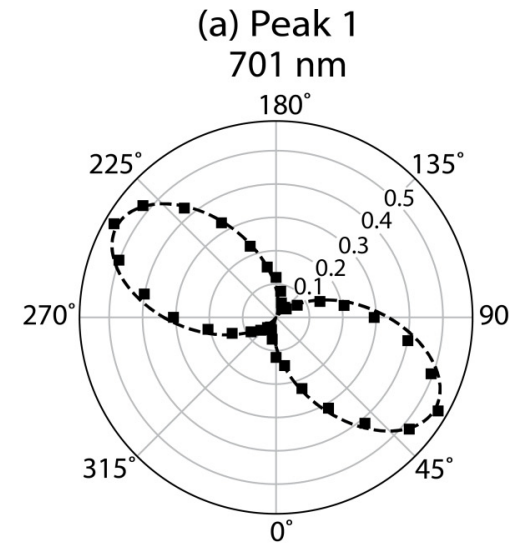

(d) Peak 4 $539 \mathrm{~nm}$

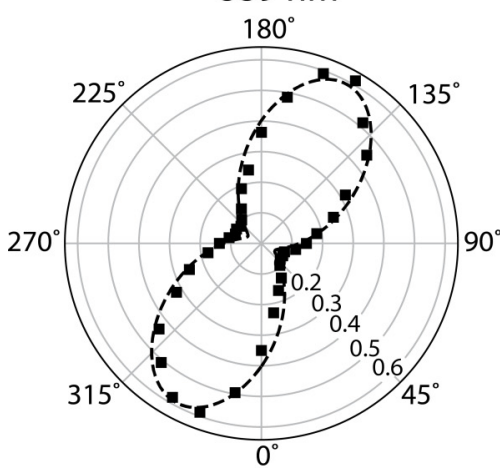

(b) Peak 2

$653 \mathrm{~nm}$

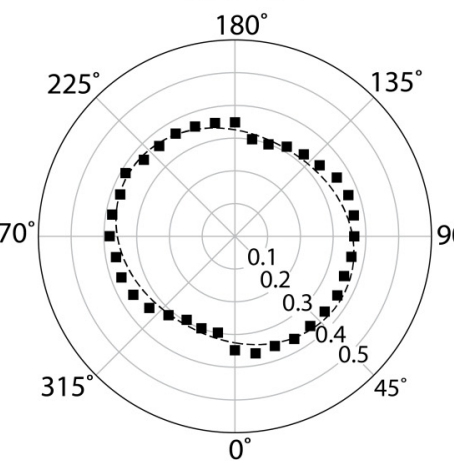

(e) Peak 5 $449 \mathrm{~nm}$

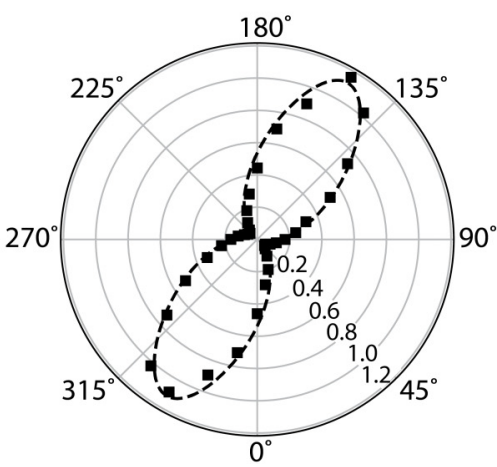

(c) Peak 3 $588 \mathrm{~nm}$

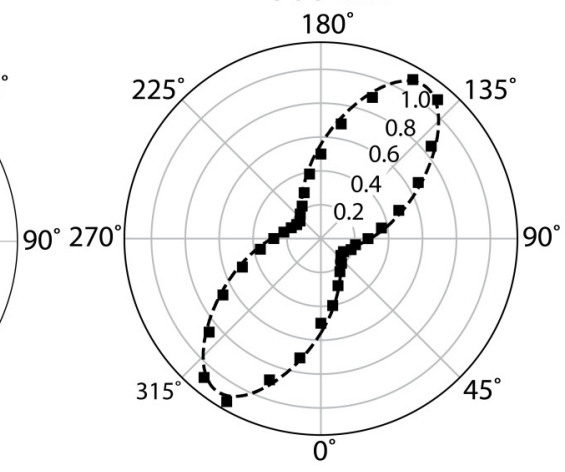

(f) Peak 6 $426 \mathrm{~nm}$

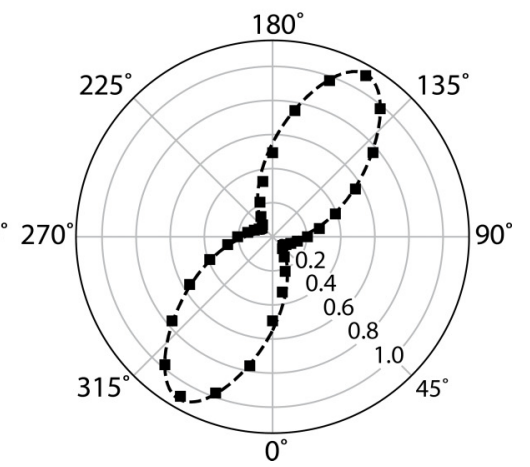

FIG. 4. (a)-(f) Polar plots of absorbance as a function of polarization angle from peak 1 to peak 6 . The dashed lines are the fitting curves and the fitting results are given in Table S1. The fitted peak positions were given. A peak shift has been observed for peak 2 and 3 as the polarization is changed from along the short axis of the molecule to the long axis of the molecule. 
(a)

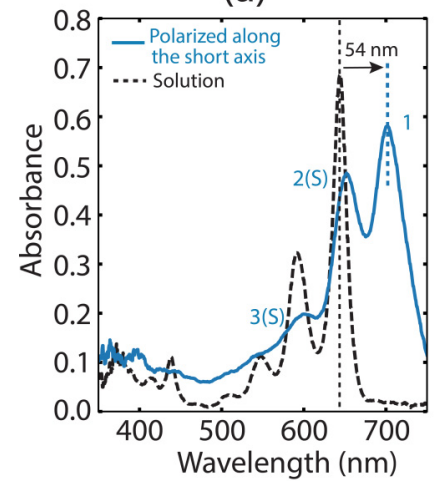

(b)

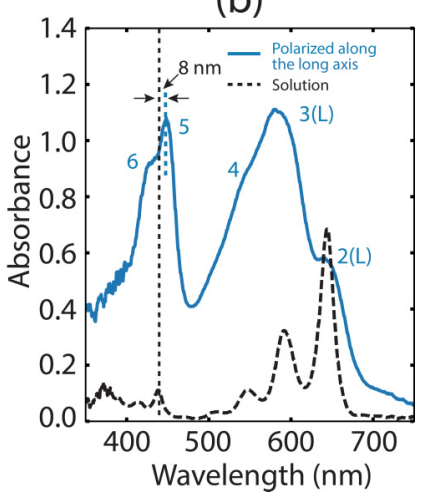

FIG. 5. (a),(b) Polarized absorption spectra along the short axis and along the long axis of a sample made at $25^{\circ} \mathrm{C}$. The sample A2 thickness is $620 \mathrm{~nm}$ and data is collected at $25^{\circ} \mathrm{C}$. The dashed line is the absorption spectrum of a dilute TIPS pentacene solution $(0.9 \mathrm{mg} / \mathrm{ml}$, toluene is the solvent).

pentacene, but this is a small perturbation that results in weak optical activity for polarization along other directions $[42,43]$. In Fig. 4, we can see that the transition dipole of peak 1 is the only one that is along the short axis of the molecule and that the transition dipoles of peak 3-6 are along the long axis of the molecule. These peaks are enhanced relative to peak 1 since the long axis of the molecule is parallel to the plane of the thin film, while transition dipoles along the short axis have only a small component in the plane of the film. Peak 2 doesn't show much polarization dependence as we discuss below.

\section{Origin of the aggregation-induced spectral shift}

Polarized absorption spectra along the short axis and along the long axis of molecules within a single grain are shown in Fig. 5. Three peaks can be observed in Fig. 5(a) which are labeled as peak 1, 2(S), 3(S). In Fig. 5(b), five peaks can be observed and are labeled as Peak 2(L), 3(L), 4, 5, 6. The absorption spectrum of a TIPS pentacene solution $(0.9 \mathrm{mg} / \mathrm{ml}$ in toluene) is plotted as a dashed line in Fig. 5. It has five absorption peaks at $643 \mathrm{~nm}, 593 \mathrm{~nm}, 550 \mathrm{~nm}, 438 \mathrm{~nm}$, and $415 \mathrm{~nm}$. The first three peaks are the Frank-Condon series of the first excited singlet state $\left(S_{0}-S_{1}\right.$ excitation).

Returning to the description of the thin film spectra, the transition dipole of peak 1 is the only one that is found to be along the short axis of the molecule. Figure 5(a) also shows that it has a large redshift relative to the solution state. Peaks $2(\mathrm{~S})$ and 2(L) are very close in position and have similar absorbance magnitudes. We note that the polar plot of peak 2 shown in Fig. 4 is a mixture of these two, so it doesn't exhibit much polarization dependence. Peak 3 is also a mixture of peak 3(S) and peak 3(L). However, since peak 3(S) has a much smaller absorbance compared to 3(L), the polar plot of peak 3 still exhibits fairly strong polarization dependence. Peaks 5 and 6 of the solid film have absorption peaks around $440 \mathrm{~nm}$, very close to two corresponding peaks in solution, indicating that they probably have the same origin. Absorbance in the short-axis polarized spectrum is significantly reduced relative to the long axis spectrum. For example, the peak $1 /$ peak 5 intensity ratio is weak in the solid-film spectrum relative to the solution spectrum. This is because the tilt angle of TIPS-pentacene molecule in the thin film is about $10^{\circ}$, thus the transition dipole along the short axis of the molecule only has a small component $\left(\sin 10^{\circ}\right)$ in the plane of the thin film, causing the peak intensity to be attenuated to only $0.03\left(\sin ^{2} 10^{\circ}\right)$ of its full value.

Our experimental results are in substantial agreement with a first-principles many-body perturbation study of quasiparticle excitations based on the GW approximation by Sharifzadeh et al. [30]. They show that peak 1 (originating from state $S_{1}$ in their notation) couples strongly to light polarized along the molecule's short axis and the rest of the peaks come from a combination of multiple excited states. They consider peak 2 to be caused by three nearly-degenerate states $\left(S_{2}, S_{3}\right.$, and $\left.S_{4}\right)$, which result from the long-range order and $\pi$ orbital overlap in the organic crystal. Optical transitions to $S_{2}$ and $S_{3}$ have the same polarization dependence as $S_{1}$, while $S_{4}$ is exactly out of phase (this is consistent with the weak angular dependence for peak 2 in Fig. 4). Based on their calculations, peaks 3 and 4 in our notation come from a mixture of numerous states. This can explain why the widths of both peaks 3 and 4 are larger than the widths of peaks 1 and 2 (Supplemental Material Table S1) [36]. For peaks 3 and 4 in our data, the net transition dipole moment is mainly aligned with the molecular backbone, so they both exhibit strong angular dependence and reach maximum absorbance when light is polarized along the long axis of the molecule. This detail doesn't agree with Sharifzadeh's calculation, which predicts that peaks 3 and 4 should be maximized when light is polarized along the short axis of the molecule [30]. Peaks 5 and 6 also have large $A_{\max } / A_{\min }$ ratios, which suggests that the transitions contributing to these two peaks are also mainly allowed along the long axis of the molecule, which is in agreement with Sharifzadeh's results [30].

In contrast to the good agreement with ab initio calculations discussed above, we note that the prediction of the optical peak shift in solid films based on simple Coulomb coupling does not explain our experimental results for the lowest energy transition (peak 1). According to Kasha et al. [44], the Coulomb coupling $J_{\text {Coul }}$ comes from the dipole interactions between the transition dipoles which can be estimated by a point-dipole approximation:

$$
J_{\text {Coul }} \approx \frac{\mu^{2}\left(1-3 \cos ^{2} \theta\right)}{4 \pi \varepsilon R^{3}},
$$

where $\mu$ is the transition dipole moment, $R$ is the intermolecular distance, $\theta$ is the angle between $\mu$ and $R$, and $\varepsilon$ is the optical dielectric constant of the medium. Equation (2) predicts $J_{\text {Coul }}>0$ for $\theta>54.7^{\circ}$. We note that more accurate treatments of the Coulomb coupling based on atomic transition charge densities give a qualitatively similar picture [45-47]. In the TIPS-pentacene crystal, the transition dipoles along the short axis of the molecular backbone are "side-byside" oriented ( $\theta$ is about $80^{\circ}$ for both dimer I and dimer II). Therefore, peak 1 is expected to be blueshifted since $J_{\text {Coul }}$ is positive, i.e., our results show that Coulomb coupling predicts the wrong direction for the shift of peak 1 in Fig. 5(a).

It has been pointed out by Spano and co-workers that the intensity ratio of the lowest energy absorption peak to its first 
TABLE II. Lattice constants of single TIPS-pentacene crystal at $23^{\circ} \mathrm{C}$ and thin films deposited at $25^{\circ} \mathrm{C}$ (sample $\left.\mathrm{B} 1 \mathrm{a}\right)$ and at $134^{\circ} \mathrm{C}$ (sample $\mathrm{B} 1 \mathrm{k})$.

\begin{tabular}{lccccccc}
\hline \hline Phase (Temp. $\left.{ }^{\circ} \mathrm{C}\right)$ & $\mathrm{a}(\AA)$ & $\mathrm{b}(\AA)$ & $\mathrm{c}(\AA)$ & $\alpha(\mathrm{deg})$. & $\beta(\mathrm{deg})$. & $\gamma(\mathrm{deg})$. & $b \sin \gamma(\AA)$ \\
\hline Bulk (23) & 7.7325 & 7.7656 & 16.9395 & 88.544 & 77.922 & 82.264 \\
Thin film I (25) & 7.78 & 7.75 & 16.70 & 89.4 & 77.9 & 81.1 & 7.6949 \\
Thin film II (134) & 8.69 & 7.66 & 16.83 & 87.8 & 78.9 & 71.0 & 7.24 \\
\% Difference & & & & & -5.5 \\
\hline \hline
\end{tabular}

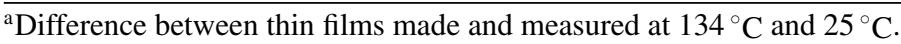

vibronic replica $(0-0 / 0-1)$ is a more reliable signature of the sign and magnitude of the excitonic coupling than the peak shift since other phenomena may dominate the peak shift. Taking the $1(\mathrm{~s}) / 2$ (s) ratio in Fig. 5 of $\approx 1.25$ as the $0-0 / 0-1$ ratio we see that it is significantly diminished compared to the corresponding ratio in the solution spectrum $(\approx 2.2)$. A reduced ratio indicates $\mathrm{H}$-type coupling $(J>0)$ in agreement with our expectation from the packing geometry. However, the magnitude of the redshift is quite large $(54 \mathrm{~nm}$, or in energy units $146 \mathrm{meV}$ ), while as we noted above there should be a blueshift for pure H-type Coulomb coupling. This contradiction can be resolved since H-type behavior in combination with a large solution to crystal redshift is a characteristic of mixing between Frenkel and charge transfer (CT) excitons in the absence of strong Coulomb coupling [22]. Although this situation may seem unnatural, such behavior has been found to be a fairly accurate description for several acenes, including tetracene and pentacene [48]. In this case, the strength of the coupling is characterized by the charge transfer coupling $J_{\mathrm{CT}}$, with $J_{\mathrm{CT}}>0$ for H-type and $\left|J_{\mathrm{CT}}\right| \gg\left|J_{\text {Coul }}\right|$.

In Sec. III C we discuss how charge transfer plays a dominant role in determining the spectral shift of peak 1. First, in Sec. III B we discuss enhancement in the hole transport.

\section{B. Tuning the charge carrier transport}

In the previous section, we determined the molecular packing geometry in the twin grains and the directions of transition dipoles of optical excitations. In this section, we show that the large anisotropic thermal expansion of TIPS-pentacene greatly affects the film's charge transport properties.

\section{Anisotropic thermal expansion in TIPS-pentacene}

Strain-free polycrystalline TIPS-pentacene thin films (samples $\mathrm{B} 1 \mathrm{a}$ and $\mathrm{B} 1 \mathrm{k}$ ) were made at $25^{\circ} \mathrm{C}$ and $134{ }^{\circ} \mathrm{C}$ to study the thermally-driven evolution of the structure. The films were deposited at high writing speed to obtain polycrystalline films, ensuring that there are enough diffraction peaks to solve for the unit cell parameters. X-ray scattering images for these two samples are shown in Figs. 6(a) and 6(b). The unit cell parameters of samples B1a and B1k were calculated from the $(11 \mathrm{~L}),(10 \mathrm{~L})$, and $(01 \mathrm{~L})$ peak positions and the results are listed in Table II. We refer to the phase made at room temperature as form I and the phase obtained above the phase transition as form II. From form I to form II, the (10L) and (11L) peaks are shifted to lower $Q_{x y}$ values whereas the $(01 \mathrm{~L})$ peaks shifted to higher $Q_{x y}$ values. We noticed the (102) reflection splits into two for form II. This is because the $Q_{z}$ of (101) and (102) for form I are 0.56 and $0.59 \AA$, respectively, which makes them difficult to distinguish, while for form II the $Q_{z}$ of (101) and (102) change to 0.53 and $0.61 \AA$, so that they are clearly separated.

From $25^{\circ} \mathrm{C}$ to $134^{\circ} \mathrm{C}$, the unit cell $a$ axis is expanded by more than $10 \%$ and the product $b \sin \gamma$ is reduced by more than $5 \%$. This result agrees well with our previous study of form I and strain-stabilized form II thin films with smaller thickness $(30 \mathrm{~nm})$ [33], indicating the structure in that study is determined by the deposition temperature and the films are strain stabilized at their high temperature lattice constants as they are cooled to room temperature due to the constraint of the substrate. In the present cases, the lattice constants are measured in situ at the deposition temperature so that strain effects are negligible. The crystallographic $d$ spacings for films made at $25^{\circ} \mathrm{C}$ and $134{ }^{\circ} \mathrm{C}$ were calculated from the unit cell parameters in Table II and are shown in Supplemental Material Table S2 [36]. As we have mentioned, the (110) $d$ spacing has the biggest change which explains why cracks tends to occur along (110) plane when cooling a thick high-temperature made sample to room temperature (Fig. 2).

Table II also includes the bulk structure from single crystal $\mathrm{X}$-ray diffraction performed at $23^{\circ}$. It is not surprising that unit cell parameters of the film deposited at $25^{\circ}$ are very similar to those of the bulk phase at $23^{\circ}$. (a)

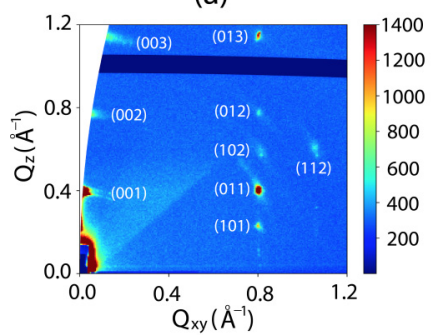

(c)

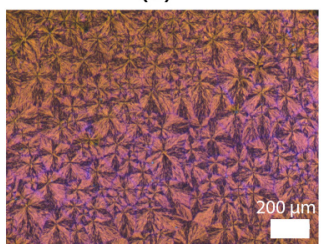

(b)

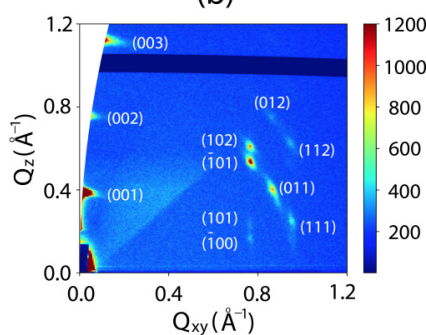

(d)

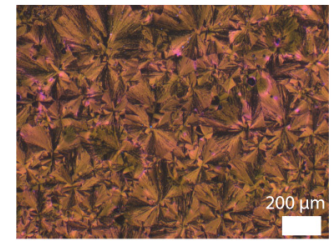

FIG. 6. (a),(b) X-ray scattering images for TIPS-pentacene films deposited at $25^{\circ} \mathrm{C}$ (sample B1a) and $134{ }^{\circ} \mathrm{C}$ (sample B1k), respectively. (c),(d) the polarized optical images of samples B1a and B1k showing that they have a spherulitic grain structure. The optical images were taken after the samples cooled to room temperature. 
(a)

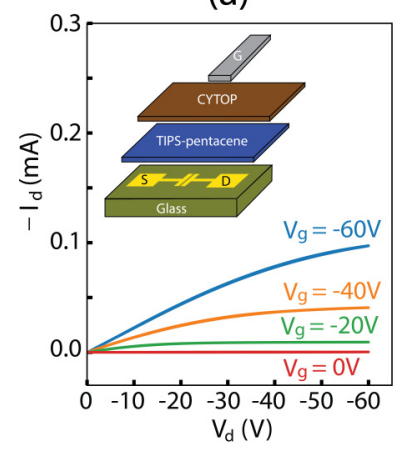

(c)

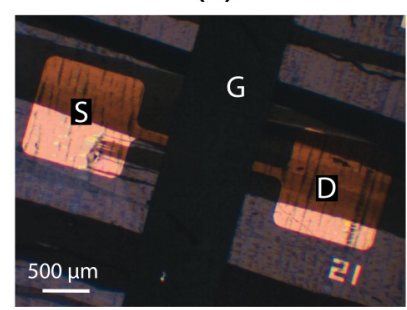

(b)

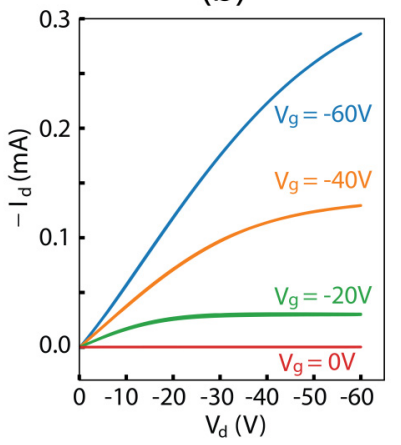

(d)

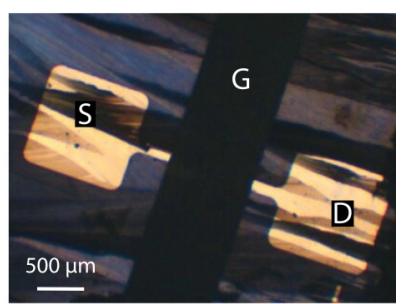

FIG. 7. (a) Output curve of a typical TIPS-pentacene based transistor using form I film as the active layer (sample B2). The inset is the exploded view of the top gate bottom contact transistor using a TIPS-pentacene film as the active layer and CYTOP film as dielectric layer. (b) Output curve of a typical TIPS-pentacene based transistor using form II film as the active layer (sample B3); (c) polarized optical image of the form I based transistor that goes with (a); (d) polarized optical image of the form II based transistor that goes with (b).

\section{Impact of structure changes on hole transport}

In order to study the charge transport properties of form II, we deposited thin TIPS-pentacene film (sample B3) at high temperature and stabilized the high temperature structure to room temperature by strain stabilization $[32,33]$. The film deposited at high substrate temperature become strained during cooling due to the mismatch between the thermal expansion coefficients of the TIPS-pentacene film and the wafer substrate. Since strain energy is proportional to film thickness, thicker films crack during cooling and release strain; this allows the lattice constants to relax to nearly strainfree values during cooling to room temperature. On the other hand, thinner films can accommodate a higher strain. Thus, the cracking temperature is depressed further and further as the film thickness is reduced. By making the Form II film thin enough, no cracks occur and the high temperature unit cell constants can be fully strain stabilized to ambient temperature [33].

The hole mobility of TIPS-pentacene form I (sample B2) and strain stabilized form II (sample B3) have been measured at room temperature by making top gate bottom contact thin film transistors using either TIPS-pentacene form I or strain stabilized form II films as active layers. The transistor geometry is shown in the inset of Fig. 7(a). The output curves of a form I based transistor and form II based transistor are shown in Figs. 7(a) and 7(b). The form I film and strain-stabilized form II morphology are shown in Fig. 7(c) and Fig. 7(d).
Crystalline grains are observed to be much longer along the writing direction than the transistor channel length $(30 \mu \mathrm{m})$. Figures 7(a) and 7(b) show that the drain current $I_{d}$ increases linearly as increasing the drain voltage $V_{d}$ in the linear region $\left(V_{d} \ll V_{g}\right)$, indicating the contact resistance between the form I or form II film and PFBT treated gold pattern is small. The form II based transistors have higher $I_{D}$ under the same gate voltage compared to form I based transistor, indicating the form II film has higher hole mobility.

The field effect hole mobilities are calculated from transfer curves in both saturation and linear region and the results are summarized in Fig. 8. Typical saturation transfer curves for TIPS-pentacene form I and form II films are shown in Figs. 8(a) and 8(b). The saturation mobility for the form I transistor is $1.0 \mathrm{~cm}^{2} \mathrm{~V}^{-1} \mathrm{~s}^{-1}$ and the saturation mobility for TIPS-pentancene form II is $3.5 \mathrm{~cm}^{2} \mathrm{~V}^{-1} \mathrm{~s}^{-1}$. The gate voltage dependence of the saturation mobility [Fig. 8(c)] does not have a pronounced mobility-overestimation peak due to nonlinear charge injection, which is an experimental artifact frequently found in the literature [49]. The linear transfer curves for the same transistors are given in Fig. 8(d) and the gate voltage dependence of the linear mobility is shown in Fig. 8(e). For the form I based transistor, its linear mobility is $0.9 \mathrm{~cm}^{2} \mathrm{~V}^{-1} \mathrm{~s}^{-1}$ which is almost equal to its saturation mobility. The linear mobility for the form II based transistor is about $2.9 \mathrm{~cm}^{2} \mathrm{~V}^{-1} \mathrm{~s}^{-1}$ which is also close to its saturation mobility.

The average linear mobility of TIPS-pentacene form I is $0.8( \pm 0.1) \mathrm{cm}^{2} \mathrm{~V}^{-1} \mathrm{~s}^{-1}$. It is close to its average saturation mobility which is $0.9( \pm 0.1) \mathrm{cm}^{2} \mathrm{~V}^{-1} \mathrm{~s}^{-1}$. The average threshold voltage is $-2 \mathrm{~V}$ and on/off ratio is about $10^{4}$. For stain-stabilized form II, the average linear and saturation mobility are $2.6( \pm 0.3) \mathrm{cm}^{2} \mathrm{~V}^{-1} \mathrm{~s}^{-1}$ and $2.9( \pm 0.5) \mathrm{cm}^{2} \mathrm{~V}^{-1} \mathrm{~s}^{-1}$ respectively. The average threshold voltage is $-3 \mathrm{~V}$ and on/off ratio is also about $10^{4}$. Therefore the mobility is increased by about a factor of three using a strained film as the active layer.

\section{Sensitivity of charge transfer integrals to the dynamic disorder}

TIPS-pentacene has two distinct dimers with a relatively large molecular orbital overlap. Dimer I is along the [100] direction and dimer II is along the [110] direction. The structure files of TIPS-pentacene form I and form II are given in the Supplemental Material [36]. A schematic of dimer I and dimer II for TIPS-pentacene form I and form II are shown in Fig. 9(a). The charge transfer integrals in a dimer depend on the nodal structure of highest occupied and lowest unoccupied molecular orbitals and the relative displacement of neighboring molecules. Varying the temperature causes neighboring molecules to move relative to each other, leading to a large modulation of charge transport properties. We consider a dimer made of two TIPS-pentacene molecules and study the variation of the charge transfer integral when one molecule "slides" with respect to the other on parallel planes with a constant separation of $3.4 \AA$, which is a good approximation of the structure change at various temperatures from experimental results. The direction along the long axis of the molecule is defined as the $x$ axis and the direction along the short axis is defined as the $y$ axis. The map of $t_{h}$ and $t_{e}$ are 
(a)

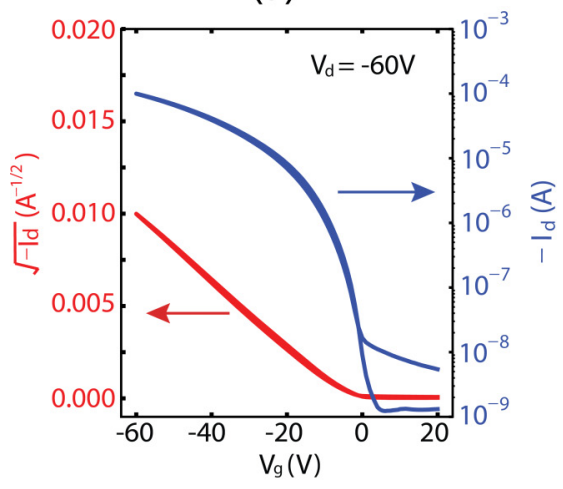

(d)

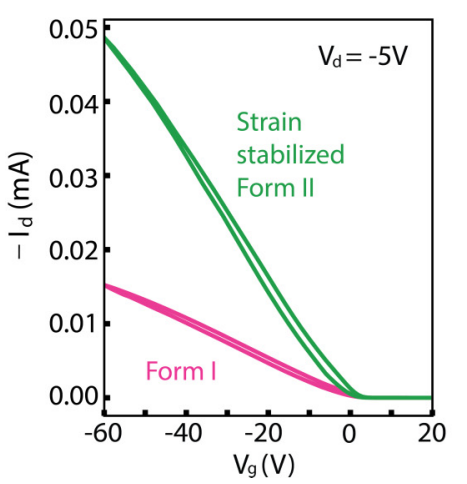

(b)

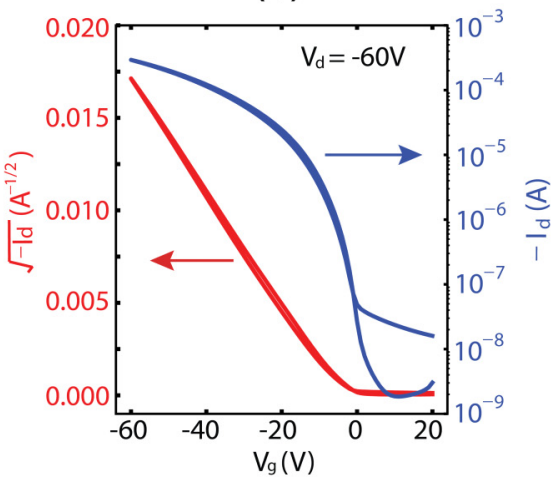

(c)

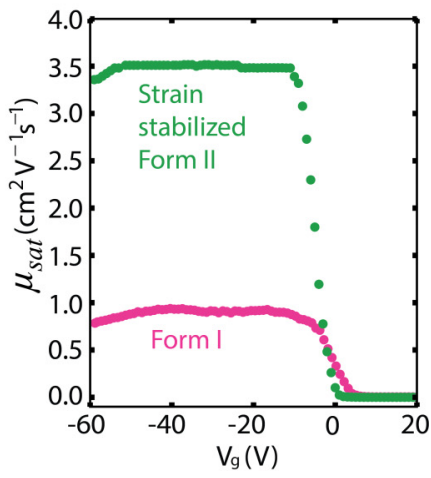

(e)

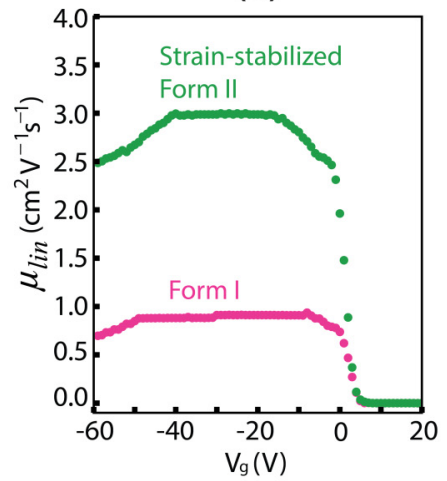

FIG. 8. (a) Saturation transfer curve for form I film made at $25^{\circ} \mathrm{C}$ (sample B2); (b) saturation transfer curve for form II film made at $130{ }^{\circ} \mathrm{C}$ and then strain stabilized to $25^{\circ} \mathrm{C}$ (sample B3); (c) gate dependent saturation mobilities for form I film and form II film; (d) linear transfer curves for form I and II film; (e) gate dependent linear mobilities for form I and form II film. The saturation and linear transfer curves were measured for the same transistor and the output curves are given in Fig. 7.

(a)

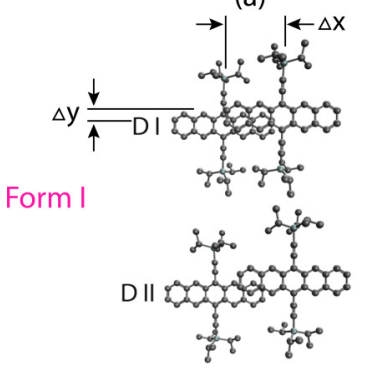

(b)

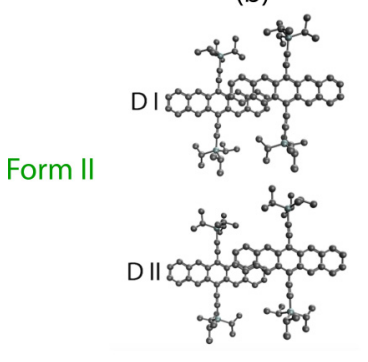

(c) $t_{h}$

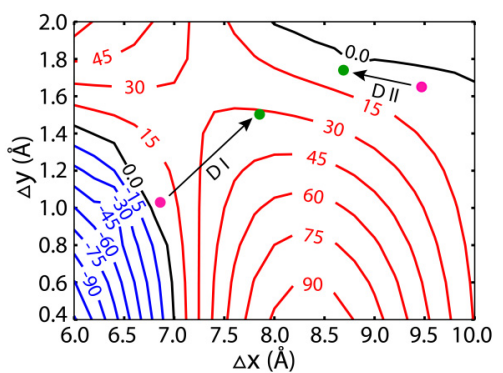

(d) te

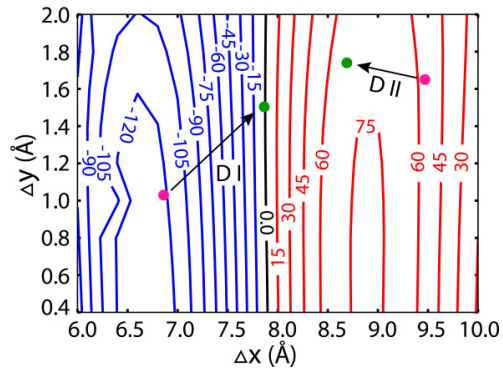

FIG. 9. (a) Schematic of dimer I and II for form I; (b) schematic of dimer I and II for form II; (c) the map of hole charge transfer integral $t_{h}$; (d) the map of electron transfer integral $t_{e}$. Magenta circles are the dimer I and II positions for Form I and green circles are the positions for form II.

shown in Figs. 9(c) and 9(d) where $t_{h}$ and $t_{e}$ are the hole and electron transfer integrals, respectively.

The sign and magnitude of $t_{h}$ and $t_{e}$ are found to be highly sensitive to the relative positions of the molecules in the dimer [50]. The $t_{h}$ and $t_{e}$ are calculated using the $\Delta x, \Delta y$, and $\pi-\pi$ stacking distance obtained from the structure files of form I and form II which are given in Table III. In references, the transfer integrals in TIPS-pentacene $[50,51]$ are usually calculated using a low temperature structure obtained at $-100{ }^{\circ} \mathrm{C}$ [52]. This low temperature phase has its $a$ lattice constant equal to $7.5650 \AA$. This parameter is different than our room temperature bulk form I structure, which has $a=7.7325 \AA$. The $\Delta x$ and $\Delta y$ for the dimer I of the $-100{ }^{\circ} \mathrm{C}$ structure are $6.70 \AA$ and $0.89 \AA$, respectively, which are slightly smaller than our form I phase whose $\Delta x$ and $\Delta y$ are $6.86 \AA$ and $1.03 \AA$, respectively. Even though this low temperature structure is similar to our room temperature phase, it still leads to noticeably different charge transfer integrals. For example, the calculated $t_{h}$ for dimer I using our room temperature form I phase is $2.6 \mathrm{meV}$ but it is $-23.5 \mathrm{meV}$ for the low temperature structure.

The unit cell of form II is obtained by $\mathrm{x}$-ray diffraction from sample B1k and the molecular packing geometry is obtained by DFT energy minimization with constrained lattice constants (results in Table II). From form I to form II, the $\Delta x$ and $\Delta y$ displacements increased for dimer I. For dimer II, the $\Delta x$ decreased while the $\Delta y$ increased. We noticed that for our geometry optimized form II, the $\pi-\pi$ stacking distances for 
TABLE III. Calculated hole and electron transfer integrals.

\begin{tabular}{|c|c|c|c|c|c|c|c|}
\hline Temp. ${ }^{\circ} \mathrm{C}$ & Dimer & $\Delta x(\AA)$ & $\Delta y(\AA)$ & $\pi-\pi(\AA)$ & $t_{h}(\mathrm{meV})$ & $t_{e}(\mathrm{meV})$ & $t_{h}+t_{e}(\mathrm{meV})$ \\
\hline 23 & Dimer I & 6.86 & 1.03 & 3.41 & 2.6 & -121.2 & -118.6 \\
\hline 134 & Dimer I & 7.87 & 1.49 & 3.36 & 33.4 & -0.3 & 33.1 \\
\hline 23 & Dimer II & 9.47 & 1.65 & 3.39 & 4.0 & 56.8 & 60.8 \\
\hline 134 & Dimer II & 8.68 & 1.77 & 3.52 & 5.7 & 56.4 & 62.1 \\
\hline
\end{tabular}

both dimers are still around 3.4-3.5 $\AA$. This is different from $\mathrm{X}$-ray diffraction results in the literature, which suggest that form II dimer I has a much smaller $\pi-\pi$ stacking distance (3.23 $\AA$ ) compared to the $\pi-\pi$ stacking distance of form II dimer II (3.65 $\AA$ ) [53]. We note that the results in the literature were based on a small number of x-ray reflections $(N=30)$, which leads to larger uncertainties in the structural parameters compared to typical single-crystal x-ray results [27,52,54].

The large hole mobility of form II strain stabilized thin films can be attributed to the increased hole charge transfer integral along dimer I and the reduced sensitivity of the charge transfer integral to dynamic disorder. It was reported that TIPS-pentacene has large thermal dynamic disorder due to molecular vibrations along the long axis of the molecule $[55,56]$. The dynamic disorder localizes the charge carriers and is detrimental to charge transport. Based on the transient localization model, reducing the sensitivity of the charge transfer integral to the dynamic disorder or suppressing the dynamic disorder are more effective ways to improve the charge mobility than by just increasing the absolute value of charge transfer integral [4]. In Fig. 9(c), we can see that the $t_{h}$ of form I is highly sensitive to the molecular vibration along the long axis of the molecule since the gradient of the hole mobility along the $x$ direction at the magenta dot in Fig. 9(c) is relatively large. On the other hand, the $t_{h}$ of Form II is less sensitive to thermal motion since it sits near a saddle point (green dot). Thus the increased mobility along form II dimer I can be partially attributed to the reduced sensitivity of charge transport to dynamic lattice disorder.

To carry this discussion further, we can estimate the transient localization length of hole carriers from our experimentally measured hole mobility and compare the difference between TIPS-pentacene form I and form II. Based on the transient localization model, the analytical formula for the charge mobility is given by [4]:

$$
\mu=\frac{e}{k_{B} T} \frac{L(\tau)^{2}}{2 \tau},
$$

where $k_{B}$ is the Boltzmann constant, $T$ is the temperature, $e$ is the electric charge, $\tau$ is the fluctuation time given by the inverse of typical intermolecular oscillation frequency, and $L(\tau)$ is the transient localization length that depends on charge transfer integral and its sensitivity to thermal vibration. The fluctuation time $\tau$ is given to be 0.15 ps for TIPS-pentacene [4]. Utilizing our experimentally measured average saturation mobility of form I which is $0.85 \mathrm{~cm}^{2} \mathrm{~V}^{-1} \mathrm{~s}^{-1}$ one can estimate the corresponding transient localization length for form I. The result is $L=8.1 \AA$, which is only slightly larger than the lattice constant of form I whose $a=7.78 \AA$. Similarly the transient localization length for form II is estimated as $15.0 \AA$ for its average saturation mobility of $2.9 \mathrm{~cm}^{2} \mathrm{~V}^{-1} \mathrm{~s}^{-1}$. The suppressed transient localization length of form I relative to form II is attributed to both a small charge transfer integral and large sensitivity to dynamic disorder as illustrated in Fig. 9(c) and reported in several theoretical studies [4,57].

\section{Tuning the optical excitation energies}

\section{Impact of structural changes on the optical absorbance}

Results of a study of the temperature dependence of absorption are shown in Fig. 10. The polarized absorption spectra along both the long and short axes were collected by heating a thick TIPS-pentacene film (sample C1). The absorption peak shift caused by the reversible temperaturedependent structure evolution is gradual and continuous. Due to the large thickness $(650 \mathrm{~nm})$, the film has cracks that allow it to keep adjusting to its equilibrium lattice constants as the temperature changes. We observe that peak 1 in Fig. 10(a) exhibits a total blueshift of about $25 \mathrm{~nm}$ between $30^{\circ} \mathrm{C}$ and $140^{\circ} \mathrm{C}$ (from $695 \mathrm{~nm}$ to $670 \mathrm{~nm}$ ).

The large sensitivity of the peak positions to temperature is consistent with the modulation of charge transfer effects as the structure changes due to thermal expansion. We note that a theoretical study of the electron-hole correlation function for the first four excited states in crystalline TIPS-pentacene has been carried out by Sharifzadeh et al. [30]. In that study, the electron-hole correlation for the low energy excitation corresponding to peak 1 is found to be mainly along the direction that corresponds to dimer I. This result helps to tie together our experimental observations, since we found in Sec. III A that there is a large change in $\Delta x$ and $\Delta y$ in dimer I (a)

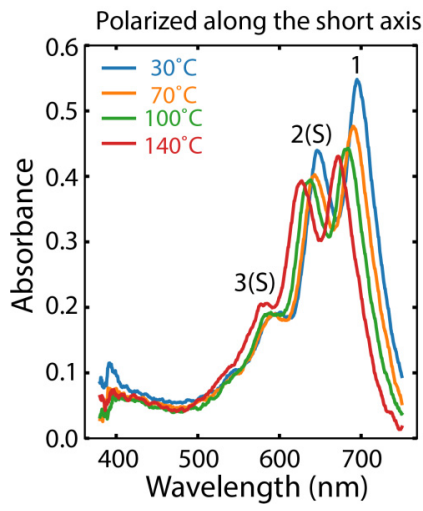

(b)

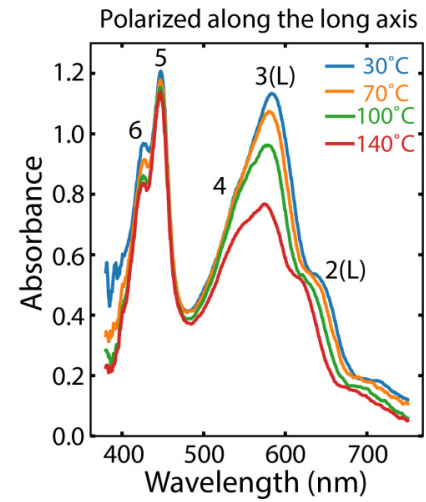

FIG. 10. The temperature dependence of polarized absorption spectra collected when a $650 \mathrm{~nm}$ thick TIPS-pentacene film was annealed from $30^{\circ} \mathrm{C}$ to $140{ }^{\circ} \mathrm{C}$ : (a) The polarization direction is along the short axis of the molecules; (b) the polarization direction is along the long axis of the molecules. 
(a)

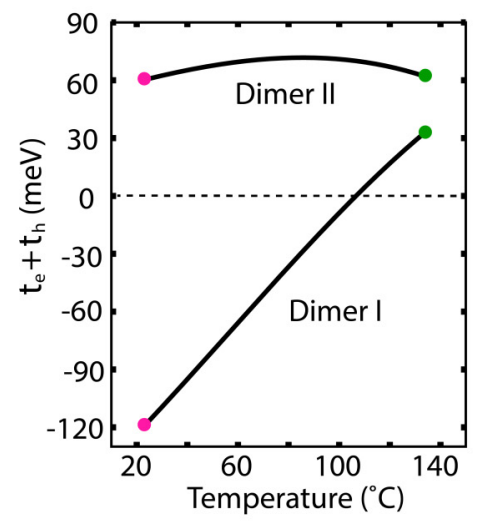

(b)

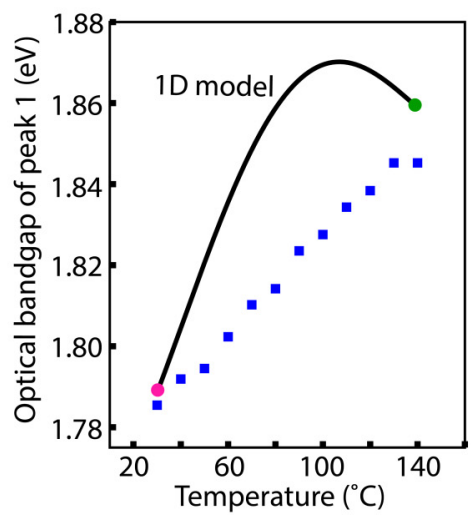

(c)

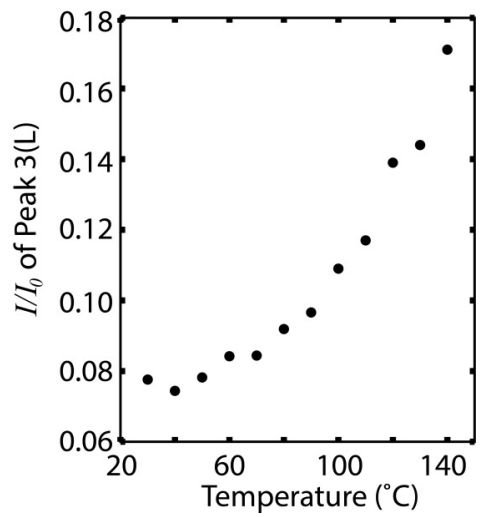

FIG. 11. (a) The change of $t_{h}+t_{e}$ for TIPS-pentacene dimer I and dimer II as the structure changes from form I to form II. (b) The optical band-gap shift of peak $1 \mathrm{vs}$ thermally-driven structure evolution. The blue squares are the experimental data (sample C1 in Table I) and the black curve is based on the 1D Frenkel/CT exciton mixing model using $t_{h}+t_{e}$ for dimer I only. (c) The temperature dependence of peak 3 (L) intensity on linear scale $I / I_{0}$.

as the temperature is changed, while in Sec. III B we found that these shifts produce a large modulation of the charge transfer integrals $t_{e}$ and $t_{h}$ for dimer I. In the next subsection, we discuss a semiquantitative one-dimensional (1D) chain model, which gives insight on how the excitation energies depend on the charge transfer integrals.

\section{Charge transfer effect on the lowest optical excitation energy}

In this section we discuss the structure sensitivity of the lowest energy optical excitation energy (peak 1). In the solid state, there are several factors that can affect the optical excitation energy including: (i) Coulombic coupling and (ii) Frenkel/CT exciton mixing $[22,44]$. As we have discussed above, Coulombic coupling does not explain the shift from solution to solid film. We find that it also fails to explain the temperature dependence since from form I to form II the Coulombic coupling is expected to be weakened as the molecular centers move further apart. This should cause peak 1 to redshift, which is inconsistent with our experimental results for the temperature dependence shown in Fig. 10(a).

The Frenkel/CT exciton mixing can change the lowest optical excitation energy in high mobility materials since their frontier orbitals have significant orbital overlap. This effect provides the most plausible explanation for the temperaturedependent peak shift. A simple 1D model can be used to semiquantitatively illustrate how Frenkel/CT exciton mixing affects the excitation energy. The Frenkel and CT excitons are coupled via the electron and hole transfer integrals $t_{e}$ and $t_{h}$ $[12,58]$. Since the CT exciton energy is typically higher than the energy of the Frenkel exciton, $E_{\mathrm{CT}}$ will be repelled upward and $E_{F}$ will be repelled downward by an amount governed by $\left|t_{h}+t_{e}\right|[22,25]$. The energies of the resultant coupled Frenkel state $\left(E_{-}\right)$and the CT exciton state $\left(E_{+}\right)$can be expressed as [22]:

$$
E_{ \pm}=\frac{E_{\mathrm{CT}}+E_{F}}{2} \pm \sqrt{\left(\frac{E_{\mathrm{CT}}-E_{F}}{2}\right)^{2}+2\left(t_{e}+t_{h}\right)^{2}}
$$

As only the Frenkel-like energy level $E_{-}$contains significant oscillator strength, Frenkel/CT mixing causes a redshift of the excitation energy [22,58]. Table III shows that from form I to form II, $t_{h}+t_{e}$ changes significantly from $-118 \mathrm{meV}$ to $33 \mathrm{meV}$ for dimer I but is almost unchanged for dimer II, as is plotted in Fig. 11(a). We assume that changes in $\Delta x$ and $\Delta y$ are linear as the temperature increases so we can calculate the temperature dependence of $t_{h}+t_{e}$. The $E_{-}$is calculated using Eq. (4) with estimated $E_{\mathrm{CT}}=2.10 \mathrm{eV}$ and $E_{F}=$ $1.87 \mathrm{eV}$ and compared with our experimental data, as shown in Fig. 11(b). We see that the model significantly overshoots, particularly in the temperature range where $t_{e}+t_{h}$ for dimer I is close to zero $\left(E_{-}=E_{F}\right.$ when $\left.t_{e}+t_{h}=0\right)$. Therefore, the data suggests that charge transfer along dimer II cannot be neglected. For simplicity, in the model shown in Fig. 11(b) we have also neglected polarization energy changes resulting from the temperature dependence of the molecular separation. However, the modulation of $E_{-}$through this mechanism is modest compared to that produced by the variation of $\left|t_{e}+t_{h}\right|$.

A consequence of this model is that $J_{\mathrm{CT}}$ can be positive or negative depending on the signs of $t_{e} t_{h}$ and $E_{\mathrm{CT}}-E_{F}$. In the limit $\left|E_{\mathrm{CT}}-E_{F}\right| \gg\left|t_{e}\right|,\left|t_{h}\right|$, it takes the simple form $J_{\mathrm{CT}}=$ $-2 t_{e} t_{h} /\left(E_{\mathrm{CT}}-E_{F}\right)[22,26,58,59]$. Assuming $E_{\mathrm{CT}}>E_{F}$, the coupling is $\mathrm{H}$-like when $t_{e} t_{h}$ is negative, i.e., $J_{\mathrm{CT}}$ is positive. We can see in Table III that $t_{e}$ and $t_{h}$ have opposite signs for dimer I, which reinforces our experimental results that are consistent with $J_{\mathrm{CT}}>0$.

The $1(\mathrm{~s}) / 2$ (s) peak ratio changes significantly as a function of temperature in Fig. 10(a). Since the ratio is reduced as the temperature increases, the theory of Spano and co-workers predicts that the exciton bandwidth increases [59]. However, this intensity ratio change should be interpreted with caution since Sharifzadeh et al. find that peak 2(s) is unlikely to be solely the results of a vibronic progression [30]. Rather, there may be two other electronic excitations with the same polarization and at nearly the same wavelength. Thus, a bandwidth extracted from the $1(\mathrm{~s}) / 2$ (s) ratio may be highly overestimated. We have argued in Sec. IIIA3 that the reduced $0-0 / 0-1$ ratio of $\approx 1.25$ is qualitatively consistent with H-type coupling. However, here we use an alternative method to obtain a quantitative estimate of the exciton bandwidth using the 
values of $E_{\mathrm{CT}}, E_{F}, t_{e}$, and $t_{h}$ from our model and a generalized wave-vector-dependent form of Eq. (4) [22,59]. The result is that the exciton bandwidth is on the order of $10 \mathrm{meV}$ for both form I and form II. We note that this approach suggests that the maximum dispersion switches from the direction of dimer I for form I, to the direction of dimer II for form II and that it becomes $J$-like $\left(J_{\mathrm{CT}}<0\right)$.

\section{Temperature dependence of higher energy excitations}

In addition to the large wavelength shift of peak 1 , Fig. 11(c) shows a dramatic change in the intensity $I$ of peak 3(L) normalized to the incident light intensity $I_{0}$ as the temperature increases. The peak intensity ratio $I / I_{0}$ increases by a factor of 2.2, as shown in Fig. 11(c), and the absorption maximum is blueshifted from $2.120 \mathrm{eV}$ to $2.153 \mathrm{eV}$ (see Supplemental Material Fig. S2) [36]. The results shown in Fig. 5 show that the Peak 3(L) does not occur for TIPS-pentacene monomers in solution, rather it is related to crystallization. Both its peak energy and peak intensity are determined by intermolecular interactions that are sensitive to thermal expansion effects. Peak 2(L) also exhibits significant temperature dependence, although it is not clear whether it is predominantly a change of intensity or wavelength.

\section{DISCUSSION}

The $\pi-\pi$ stacking distance in molecular crystals is determined by attractive van der Waals binding in the dimer, balanced with electrostatics and exchange repulsion interactions. Since these forces are hypersensitive to the molecular separation distance, the $\pi-\pi$ stacking distance in $\pi$ conjugated materials tend to maintain at a constant value $3.4-3.5 \AA$, consistent with our combined experimental/theoretical results. This places us in a position to discuss how the TIPS-pentacene crystals mechanically respond to changes in temperature or to mechanical strain.

In the literature, it is reported that compressive strain along the $\pi-\pi$ stacking distance reduces the molecular centerto-center distance, thus enhancing the charge transport in TIPS-pentacene and PTCDI-C8 [60]. However, our results for TIPS-pentacene indicates that the $\pi-\pi$ stacking distance is nearly independent on the molecular center-to-center distance. It is likely that this same behavior can also take place when compressive or tensile strain is applied to a crystal. For molecules packed in a bricklayer stacking, molecules in a dimer can rotate under strain to keep a constant $\pi-\pi$ stacking distance as the crystal is either stretched or compressed along a particular crystallographic direction. Thus, we predict that counterintuitively, tensile strain can increase charge mobility as long as the relative molecular positions move towards larger charge transfer integrals or towards reduced sensitivity to dynamic disorder (or both, as we have observed in Fig. 9).

For form I, the transient localization length is estimated to be only slightly larger than the molecular spacing in the dimer, indicating that the field effect mobility is consistent with localized charge carriers. The Hall effect mobility has been found to be much smaller than its field effect mobility for form I, which was attributed to charge carriers being too strongly localized to contribute to a Hall voltage [15]. On the other hand for form II the transient localization length is nearly doubled, thus the charge carriers are more delocalized. These results suggest that the Hall effect mobility for form II should be close to its field effect mobility since delocalized charge carriers can couple to the magnetic field through the Lorentz force.

In this paper, our main achievement is that we have established reliable structure-property relationships for TIPSpentacene thin films based on our results on TIPS-pentacene molecular orientation, transition dipoles direction, and the field effect mobility characterization. Establishing clear structure-property relationships for TIPS-pentacene is challenging, and we have noticed that there are discrepancies and even contradictory results reported in the literature. Several of these issues include: (1) Multiple groups have reported that the twin boundary of TIPS-pentacene twin grains are along [210] directions [27,61], but our previous report [28] and others $[37,38]$ have found that the boundary is along the [100] direction. It is not yet clear whether these differences are a result of different thin film deposition conditions or due to spurious experimental results. (2) Experimental reports in the literature find that the lowest energy absorption peak of TIPSpentacene has its transition dipole along the long axis of the molecule [29], while a theoretical study finds that the transition dipole of lowest energy absorption peak is along the short axis of the molecule [30]. Our results clearly favor the latter finding. (3) A large spread of mobility values have been reported in the literature for TIPS-pentacene. For example, surprisingly high mobility has been reported for both TIPS-pentacene form I and form II. The "record" highest average mobility reported for TIPS-pentacene form I is $6.9 \mathrm{~cm}^{2} \mathrm{~V}^{-1} \mathrm{~s}^{-1}$, while for form II it is $8.1 \mathrm{~cm}^{2} \mathrm{~V}^{-1} \mathrm{~s}^{-1}$ $[62,63]$. However, reproducible values from different groups $[6,14,64]$ suggest that the TIPS-pentacene form I has a field effect mobility that is actually somewhat lower, in the range of $0.4-1.2 \mathrm{~cm}^{2} \mathrm{~V}^{-1} \mathrm{~s}^{-1}$, which is consistent with the transient localization model [4]. It has been cautioned that overestimation of mobility could result from voltage-dependent contact resistance effects $[10,49]$. In contrast, our results follow recommended best practices in the literature, such as reporting the linear mobility as the mobility of record and ruling out gate-voltage dependence of the estimated mobility [65]. The average linear mobility for TIPS-pentacene form I is 0.8 $( \pm 0.1) \mathrm{cm}^{2} \mathrm{~V}^{-1} \mathrm{~s}^{-1}$ and for strain-stabilized TIPS-pentacene form II is $2.6( \pm 0.3) \mathrm{cm}^{2} \mathrm{~V}^{-1} \mathrm{~s}^{-1}$.

\section{CONCLUSIONS}

In this paper, we have clarified several questions related to the TIPS-pentacene molecular packing and transition dipole directions, and we have described how the structure sensitivity of charge carrier mobility and low energy optical excitations are both essentially determined by the change of the magnitude of charge transfer integrals and their gradient with respect to molecular displacement in the crystal. The hole mobility of strain-stabilized form II TIPS-pentacene is about three times higher than form I due to the increased magnitude of the hole transfer integral and reduced sensitivity to dynamic disorder. Strain engineering is a general approach to improve mobility by tuning the molecular positions in the solid towards 
positions where charge transfer integrals are large and insensitive to the molecular thermal vibrations. We anticipate that this approach can be applied to many other organic semiconductors to improve their charge carrier mobility since their structures can be tuned by a combination of thermal expansion and mechanical strain. We have also determined that the thermal expansion effect in TIPS-pentacene causes the molecules in the dimers to "slide" with respect to each other while maintaining an almost constant $\pi-\pi$ stacking distance. As a result, hole transfer is enhanced even though the molecular centers move further apart during thermal expansion, and the lowest energy absorption peak of TIPS-pentacene is blueshifted in form II due to Frenkel/CT exciton mixing. The links between optical excitation and carrier transport via charge transfer integrals highlight the combination of structural, electronic, and optical measurements with first principles theory as a powerful toolset to monitor and predict the properties of strain-engineered materials with improved carrier mobility or desirable optical properties.

\section{ACKNOWLEDGMENTS}

The authors thank Prof. John M. Hughes for his help on collecting single-crystal x-ray diffraction data, Prof. Mathew White for his assistance on film thickness measurement, and Prof. Michael Ruggiero for helpful discussions. This work is supported by the National Science Foundation under Grants No. DMR-1307017 and No. DMR-1701774. The Xray scattering measurements were conducted at the Cornell High Energy Synchrotron Source (CHESS) which is supported by the National Science Foundation under NSF award DMR-1332208.
[1] L. A. Stevens, K. P. Goetz, A. Fonari, Y. Shu, R. M. Williamson, J.-L. Brédas, V. Coropceanu, O. D. Jurchescu, and G. E. Collis, Temperature-mediated polymorphism in molecular crystals: The impact on crystal packing and charge transport, Chem. Mater. 27, 112 (2015).

[2] J. Mizuguchi and K. Tojo, Electronic structure of perylene pigments as viewed from the crystal structure and excitonic interactions, J. Phys. Chem. B 106, 767 (2002).

[3] G. Schweicher, Y. Olivier, V. Lemaur, and Y. H. Geerts, What currently limits charge carrier mobility in crystals of molecular semiconductors? Isr. J. Chem. 54, 595 (2014).

[4] S. Fratini, S. Ciuchi, D. Mayou, G. T. de Laissardiére, and A. Troisi, A map of high-mobility molecular semiconductors, Nat. Mater. 16, 998 (2017).

[5] V. Coropceanu, J. Cornil, D. A. da Silva Filho, Y. Olivier, R. Silbey, and J.-L. Brédas, Charge transport in organic semiconductors, Chem. Rev. 107, 926 (2007).

[6] Sung Kyu Park, T. N. Jackson, J. E. Anthony, and D. A. Mourey, High mobility solution processed 6,13bis(triisopropyl-silylethynyl) pentacene organic thin film transistors, Appl. Phys. Lett. 91, 063514 (2007).

[7] D. O. Hutchins, T. Weidner, J. Baio, B. Polishak, O. Acton, N. Cernetic, H. Ma, and K.-Y. Jen, Effects of self-assembled monolayer structural order, surface homogeneity and surface energy on pentacene morphology and thin film transistor device performance, J. Mater. Chem. C 1, 101 (2013).

[8] J. Wan, Y. Li, J. G. Ulbrandt, D.-M. Smilgies, J. Hollin, A. C. Whalley, and R. L. Headrick, Transient phases during fast crystallization of organic thin films from solution, APL Materials 4, 016103 (2016).

[9] K. Takimiya, I. Osaka, T. Mori, and M. Nakano, Organic semiconductors based on [1]benzothieno[3,2-b][1]benzothiophene substructure, Acc. Chem. Res. 47, 1493 (2014).

[10] A. F. Paterson, S. Singh, K. J. Fallon, T. Hodsden, Y. Han, B. C. Schroeder, H. Bronstein, M. Heeney, I. McCulloch, and T. D. Anthopoulos, Recent progress in high-mobility organic transistors: A reality check, Adv. Mater. 30, 1801079 (2018).

[11] S. Sharifzadeh, P. Darancet, L. Kronik, and J. B. Neaton, Lowenergy charge-transfer excitons in organic solids from firstprinciples: The case of pentacene, J. Phys. Chem. Lett. 4, 2197 (2013).
[12] P. Cudazzo, F. Sottile, A. Rubio, and M. Gatti, Exciton dispersion in molecular solids, J. Phys.: Condens. Matter 27, 113204 (2015).

[13] R. A. Marcus, Electron transfer reactions in chemistry: Theory and experiment, Rev. Mod. Phys. 65, 599 (1993).

[14] T. Sakanoue and H. Sirringhaus, Band-like temperature dependence of mobility in a solution-processed organic semiconductor, Nat. Mater. 9, 736 (2010).

[15] J.-F. Chang, T. Sakanoue, Y. Olivier, T. Uemura, M.-B. Dufourg-Madec, S. G. Yeates, J. Cornil, J. Takeya, A. Troisi, and H. Sirringhaus, Hall-Effect Measurements Probing the Degree of Charge-Carrier Delocalization in Solution-Processed Crystalline Molecular Semiconductors, Phys. Rev. Lett. 107, 066601 (2011).

[16] A. Troisi and G. Orlandi, Charge-Transport Regime of Crystalline Organic Semiconductors: Diffusion Limited by Thermal off-Diagonal Electronic Disorder, Phys. Rev. Lett. 96, 086601 (2006).

[17] A. Troisi and G. Orlandi, Dynamics of the intermolecular transfer integral in crystalline organic semiconductors, J. Phys. Chem. A 110, 4065 (2006).

[18] A. Troisi, Charge transport in high mobility molecular semiconductors: classical models and new theories, Chem. Soc. Rev. 40, 2347 (2011).

[19] S. Fratini, D. Mayou, and S. Ciuchi, The transient localization scenario for charge transport in crystalline organic materials, Adv. Funct. Mater. 26, 2292 (2016).

[20] P. M. Kazmaier and R. Hoffmann, A theoretical study of crystallochromy: Quantum interference effects in the spectra of perylene pigments, J. Am. Chem. Soc. 116, 9684 (1994).

[21] M. Hoffmann, K. Schmidt, T. Fritz, T. Hasche, V. M. Agranovich, and K. Leo, The lowest energy frenkel and chargetransfer excitons in quasi-one-dimensional structures: Application to MePTCDI and PTCDA crystals, Chem. Phys. 258, 73 (2000).

[22] N. J. Hestand and F. C. Spano, Molecular aggregate photophysics beyond the Kasha model: Novel design principles for organic materials, Acc. Chem. Res. 50, 341 (2017).

[23] L. Gisslén and R. Scholz, Crystallochromy of perylene pigments: Interference between Frenkel excitons and chargetransfer states, Phys. Rev. B 80, 115309 (2009). 
[24] R. W. Munn, B. Pac, and P. Petelenz, Charge-transfer-induced Frenkel exciton splitting in crystalline fullerene, Phys. Rev. B 57, 1328 (1998)

[25] M.H. Hennessy, Z.G. Soos, R.A. Pascal, and A. Girlando, Vibronic structure of PTCDA stacks: The exciton-phonon-chargetransfer dimer, Chem. Phys. 245, 199 (1999).

[26] H. Yamagata, D. S. Maxwell, J. Fan, K. R. Kittilstved, A. L. Briseno, M. D. Barnes, and F. C. Spano, H,J-aggregate behavior of crystalline 7,8,15,16-tetraazaterrylene: Introducing a new design paradigm for organic materials, J. Phys. Chem. C 118, 28842 (2014).

[27] J. Chen, C. K. Tee, M. Shtein, D. C. Martin, and J. Anthony, Controlled solution deposition and systematic study of chargetransport anisotropy in single crystal and single-crystal textured TIPS pentacene thin films, Organic Electronics 10, 696 (2009).

[28] S. Wo, R. L. Headrick, and J. E. Anthony, Fabrication and characterization of controllable grain boundary arrays in solutionprocessed small molecule organic semiconductor films, J. Appl. Phys. 111, 073716 (2012).

[29] D. T. James, J. M. Frost, J. Wade, J. Nelson, and J. S. Kim, Controlling microstructure of pentacene derivatives by solution processing: Impact of structural anisotropy on optoelectronic properties, ACS Nano 7, 7983 (2013).

[30] S. Sharifzadeh, C. Y. Wong, H. Wu, B. L. Cotts, L. Kronik, N. S. Ginsberg, and J. B. Neaton, Relating the physical structure and optoelectronic function of crystalline TIPS-pentacene, Adv. Funct. Mater. 25, 2038 (2015).

[31] R. L. Headrick, S. Wo, F. Sansoz, and J. E. Anthony, Anisotropic mobility in large grain size solution processed organic semiconductor thin films, Appl. Phys. Lett. 92, 063302 (2008).

[32] I. Cour, P. V. Chinta, C. M. Schlepütz, Y. Yang, R. Clarke, R. Pindak, and R. L. Headrick, Origin of stress and enhanced carrier transport in solution-cast organic semiconductor films, J. Appl. Phys. 114, 093501 (2013).

[33] Y. Li, J. Wan, D.-M. Smilgies, N. Bouffard, R. Sun, and R. L. Headrick, Nucleation and strain-stabilization during organic semiconductor thin film deposition, Sci. Rep. 6, 32620 (2016).

[34] G. te Velde, F. M. Bickelhaupt, E. J. Baerends, C. Fonseca Guerra, S. J. A. van Gisbergen, J. G. Snijders, and T. Ziegler, Chemistry with adf, Journal of Computational Chemistry 22, 931 (2001).

[35] J. P. Perdew, J. A. Chevary, S. H. Vosko, K. A. Jackson, M. R. Pederson, D. J. Singh, and C. Fiolhais, Atoms, molecules, solids, and surfaces: Applications of the generalized gradient approximation for exchange and correlation, Phys. Rev. B 46, 6671 (1992).

[36] See Supplemental Material at http://link.aps.org/supplemental/ 10.1103/PhysRevResearch.2.033294 for (i) two structure files corresponding to TIPS-pentacene Form I and Form II and (ii) a supplemental document containing a description of the polarized absorbance fitting method, as well as two figures and three tables of additional results.

[37] K. Sakamoto, J. Ueno, K. Bulgarevich, and K. Miki, Anisotropic charge transport and contact resistance of 6,13bis(triisopropylsilylethynyl) pentacene field-effect transistors fabricated by a modified flow-coating method, Appl. Phys. Lett. 100, 123301 (2012)

[38] K.-Y. Wu, C.-T. Hsieh, L.-H. Wang, C.-H. Hsu, S.-T. Chang, S.-T. Lan, Y.-F. Huang, Y.-M. Chen, and C.-L. Wang, Influences of out-of-plane lattice alignment on the OFET performance of TIPS-PEN crystal arrays, Crystal Growth \& Design 16, 6160 (2016).

[39] J. Chen, J. Anthony, and D. C. Martin, Thermally induced solidstate phase transition of bis(triisopropylsilylethynyl) pentacene crystals, J. Phys. Chem. B 110, 16397 (2006).

[40] A. B Djurišić, F. Torsten, and K. Leo, Modeling the optical constants of organic thin films: Application to 3,4,9,10-perylenetetracarboxylic dianhydride (PTCDA), Optics Communications 183, 123 (2000).

[41] M. Sambridge, J. F. Gerald, I. Kovács, H. St. C. O’Neill, and J. Hermann, Quantitative absorbance spectroscopy with unpolarized light: Part I. physical and mathematical development, American Mineralogist 93, 751 (2008).

[42] M. L. Tiago, J. E. Northrup, and S. G. Louie, Ab initio calculation of the electronic and optical properties of solid pentacene, Phys. Rev. B 67, 115212 (2003).

[43] C. Y. Wong, B. D. Folie, B. L. Cotts, and N. S. Ginsberg, Discerning variable extents of interdomain orientational and structural heterogeneity in solution-cast polycrystalline organic semiconducting thin films, J. Phys. Chem. Lett. 6, 3155 (2015).

[44] M. Kasha, H. R. Rawls, and M. A. El-Bayoumi, The exciton model in molecular spectroscopy, Pure and Applied Chemistry 11, 371 (1965).

[45] D. Beljonne, J. Cornil, R. Silbey, P. Millié, and J. L. Brédas, Interchain interactions in conjugated materials: The exciton model versus the supermolecular approach, J. Chem. Phys. 112, 4749 (2000).

[46] J. Gierschner and S. Y. Park, Luminescent distyrylbenzenes: Tailoring molecular structure and crystalline morphology, Journal of Materials Chemistry C 1, 5818 (2013).

[47] D. Bialas, A. Zitzler-Kunkel, E. Kirchner, D. Schmidt, and F. Würthner, Structural and quantum chemical analysis of exciton coupling in homo- and heteroaggregate stacks of merocyanines, Nat. Commun. 7, 12949 (2016).

[48] H. Yamagata, J. Norton, E. Hontz, Y. Olivier, D. Beljonne, J.-L. Brédas, R. J. Silbey, and F. C. Spano, The nature of singlet excitons in oligoacene molecular crystals, J. Chem. Phys. 134, 204703 (2011).

[49] E. G. Bittle, J. I. Basham, T. N. Jackson, O. D. Jurchescu, and D. J. Gundlach, Mobility overestimation due to gated contacts in organic field-effect transistors, Nat. Commun. 7, 10908 (2016).

[50] A. Troisi, G. Orlandi, and J. E. Anthony, Electronic interactions and thermal disorder in molecular crystals containing cofacial pentacene units, Chem. Mater. 17, 5024 (2005).

[51] J.-X. Fan, X.-K. Chen, S.-F. Zhang, and A.-M. Ren, Theoretical study on charge transport properties of intra- and extra-ring substituted pentacene derivatives, J. Phys. Chem. A 120, 2390 (2016).

[52] J. E. Anthony, J. S. Brooks, D. L. Eaton, and S. R. Parkin, Functionalized pentacene: Improved electronic properties from control of solid-state order, J. Am. Chem. Soc. 123, 9482 (2001).

[53] Y. Diao, K. M. Lenn, W.-Y. Lee, M. A. Blood-Forsythe, J. Xu, Y. Mao, Y. Kim, J. A. Reinspach, S. Park, A. Aspuru-Guzik, G. Xue, P. Clancy, Z. Bao, and S. C. B. Mannsfeld, Understanding polymorphism in organic semiconductor thin films through nanoconfinement, J. Am. Chem. Soc. 136, 17046 (2014). 
[54] H. Chung, D. Dudenko, F. Zhang, G. D’Avino, C. Ruzié, A. Richard, G. Schweicher, J. Cornil, D. Beljonne, Y. Geerts, and Y. Diao, Rotator side chains trigger cooperative transition for shape and function memory effect in organic semiconductors, Nat. Commun. 9, 278 (2018).

[55] A. S. Eggeman, S. Illig, A. Troisi, H. Sirringhaus, and P. A. Midgley, Measurement of molecular motion in organic semiconductors by thermal diffuse electron scattering, Nat. Mater. 12, 1045 (2013).

[56] S. Illig, A. S. Eggeman, A. Troisi, L. Jiang, C. Warwick, M. Nikolka, G. Schweicher, S. G. Yeates, Y. Henri Geers, J. E. Anthony, and H. Sirringhaus, Reducing dynamic disorder in small-molecule organic semiconductors by suppressing largeamplitude thermal motions, Nat. Commun. 7, 10736 (2016).

[57] S. Fratini and S. Ciuchi, Dynamical localization corrections to band transport, Phys. Rev. Research 2, 013001 (2020).

[58] H. Yamagata, C. M. Pochas, and F. C. Spano, Designing Jand $\mathrm{H}$-aggregates through wave function overlap engineering: Applications to poly(3-hexylthiophene), J. Phys. Chem. B 116, 14494 (2012).

[59] N. J. Hestand and F. C. Spano, Interference between coulombic and CT-mediated couplings in molecular aggregates: H-to Jaggregate transformation in perylene-based $\pi$-stacks, J. Chem. Phys. 143, 244707 (2015).

[60] Y. Park, K. S. Park, B. Jun, Y.-E. K. Lee, S. U. Lee, and M. M. Sung, Quantitative correlation between carrier mobility and intermolecular center-to-center distance in organic single crystals, Chem. Mater. 29, 4072 (2017).

[61] P. Fesenko, C. Rolin, R. Janneck, S. P. Bommanaboyena, H Gaethje, P. Heremans, and J. Genoe, Determination of crystal orientation in organic thin films using optical microscopy, Organic Electronics 37, 100 (2016).

[62] K. Kim, Y. Rho, Y. Kim, S. H. Kim, S. G. Hahm, and C. E. Park, A lattice-strained organic single-crystal nanowire array fabricated via solution-phase nanograting-assisted pattern transfer for use in high-mobility organic field-effect transistors, Adv. Mater. 28, 3209 (2016).

[63] Y. Diao, B. C.-K. Tee, G. Giri, J. Xu, D. H. Kim, H. A. Becerril, R. M. Stoltenberg, T. H. Lee, G. Xue, S. C. B. Mannsfeld, and Z. Bao, Solution coating of large-area organic semiconductor thin films with aligned single-crystalline domains, Nat. Mater. 12, 665 (2013).

[64] M. R. Niazi, R. Li, M. Abdelsamie, K. Zhao, D. H. Anjum, M. M. Payne, J. Anthony, D.-M. Smilgies, and A. Amassian, Contact-induced nucleation in high-performance bottom-contact organic thin film transistors manufactured by large-area compatible solution processing, Adv. Funct. Mater. 26, 2371 (2016).

[65] H. H. Choi, K. Cho, C. D. Frisbie, H. Sirringhaus, and V. Podzorov, Critical assessment of charge mobility extraction in FETs, Nat. Mater. 17, 2 (2018). 